\title{
Abundances in planetary nebulae near the galactic centre $^{\star, \star \star}$
}

\section{Abundance determinations}

\author{
M.A. Ratag ${ }^{1,2}$, S.R. Pottasch ${ }^{1}$, M. Dennefeld ${ }^{3}$, and J. Menzies ${ }^{4}$ \\ 1 Kapteyn Astronomical Institute, Groningen University, Postbus 800, 9700 AV Groningen, The Netherlands \\ 2 Indonesian National Institute of Aeronautics and Space (LAPAN), Jakarta, Indonesia \\ 3 Institut d'Astrophysique, 98bis Boulevard Arago, F-75014 Paris, France \\ 4 South African Astronomical Observatory, P.O. Box 9, Cape Town, South Africa
}

Received July 29, 1996; accepted November 18, 1997

\begin{abstract}
Abundance determinations of about 110 planetary nebulae, which are likely to be in the Galactic Bulge are presented. Plasma diagnostics have been performed by making use of the available forbidden line ratios combined with radio continuum measurements. Chemical abundances of $\mathrm{He}, \mathrm{O}, \mathrm{N}, \mathrm{Ne}, \mathrm{S}, \mathrm{Ar}$, and $\mathrm{Cl}$ are then derived by employing theoretical nebular models as interpolation devices in establishing the ionization correction factors (ICFs) used to estimate the distribution of atoms among unobserved ionization stages. The overall agreement between the results derived by using the model-ICFs and those obtained from the theoretical models is reasonably good. The uncertainties related to the total abundances show a clear dependence on the level of excitation. In most cases, the abundances of chlorine can be derived only in objects with a relatively high $\mathrm{Cl}$-abundance. Contrary to the conclusion previously drawn by Webster (1988), we found the excitation classes are not uniformly distributed. A clear peak at about classes 5 and 6 is noticed. The distribution is shifted toward a lower excitation range with respect to that of the nearby nebulae, reflecting the difference in the central star temperature distribution between the two samples.
\end{abstract}

Key words: planetary nebulae - ISM: abundances Galaxy: center

\footnotetext{
Send offprint requests to: S.R. Pottasch

* Based on observations obtained at the European Southern Observatory and at SAAO.

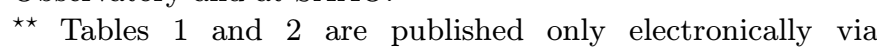
the CDS at anonymous ftp 130.79.128.5 or http://cdsweb.ustrasbg.fr/Abstract.html
}

\section{Introduction}

The study of chemical abundances and their variation in the Galaxy is of fundamental importance toward understanding galactic nucleosynthesis history. A knowledge of the variation of chemical composition across the disk has been investigated in many different ways, using various kinds of objects. Recent determinations of abundances in stars (Gehren et al. 1985; Luck 1982; Janes 1979; Pagel 1979; Mayor 1976), H II regions (Shaver et al. 1983; Peimbert et al. 1978), and planetary nebulae (Peimbert \& Torres-Peimbert 1977) reliably reveal the presence of radial gradients in galactic abundances relative to hydrogen such that metal abundances increase in the direction of galactic center. These results are consistent with the well-known electron temperature gradient indicating an increase outward from the center (Churchwell et al. 1978; Churchwell \& Walmsley 1975; Shaver et al. 1983; FaúndezAbans \& Maciel 1986). Although electron temperature, fixed by the energy balance in the corresponding system, is affected by various other factors such as energy distribution of the powering source(s), morphology and electron density, it clearly shows a negative correlation with abundances. From the theoretical point of view, the recent models of Galactic chemical evolution have been able to reproduce the observed abundance gradients of individual elements along the galactic disk (Matteucci \& François 1988; Diaz \& Tosi 1984, 1986) in spite of the large uncertainties related to a relatively large number of free parameters going into the calculations.

Abundance determinations of objects thought to be in the bulge of our Galaxy (Lester et al. 1981; Aitken et al. 1976; Price 1981; Rich 1988; Frogel \& Whitford 1987; Terndrup 1988) show an average metallicity of about two to three times solar, and thus more or less in a close agreement with the values 
extrapolated from the abundance gradients in the disk. This agreement has led to the conclusion that objects in the central bulge of our Galaxy are, on average, of extremely high metallicity. On the other hand, there is also a valid worry that such an extrapolation cannot be justified. Firstly, most objects used in establishing the abundance gradients are found at galactocentric distances greater than about $4-5 \mathrm{kpc}$ (assuming $R_{\odot}=8 \mathrm{kpc}$ ), which is still relatively far from the center. Secondly, there are indications that the galactic bulge region has a quantitatively different cycle of stellar birth and death compared with other regions in the Galaxy (Morris 1989; Wannier 1989; Güsten 1989), leading to a different nucleosynthesis history. The very different physical conditions found in the bulge are argued to be the main cause. The recent chemical evolution models for the Galactic Bulge (Rich 1986, 1990; Matteucci \& Brocato 1990) were handicapped by the lack of data on abundances of elements other than iron. Consequently, their main purpose was to fit the available $[\mathrm{Fe} / \mathrm{H}]$ distribution. While this is a necessary condition, it is certainly not sufficient, considering not only the very large number of free input parameters (e.g. the star formation rate, the initial mass function, and the infall rate) but also the indication that the estimation of iron production might be still uncertain by a factor of about 3 (Rocca-Volmerange \& Schaeffer 1990).

Planetary nebulae $(\mathrm{PNe})$ are useful probes to investigate the chemical evolution of the Galaxy. They are important from two points of view: (a) the chemical composition of the nebulae provide information on those elements which are difficult to measure in stars, and (b) they supply abundance information covering a very large range in galactocentric distance, including the region nearby the center.

It is therefore of interest to perform an extensive survey of galactic bulge PNe to see if their abundances are substantially different than those in the solar vicinity, and at the same time, whether or not they follow the trend shown by the observed abundance gradients in the disk.

The objective of this paper is to describe the abundance determinations of galactic bulge PN. This paper will be arranged as follows. In Sect. 2 the selection of objects will be discussed, including also the description of the spectral line data used in the computation. The data reduction, the classification, and the plasma diagnostics are presented in Sect. 3. Section 4 describes the procedure applied in the determination of the nebular chemical composition. The final results will be discussed in Sect. 5 . A detailed analysis of the abundances and their interpretation in terms of chemical evolution has been published (Ratag et al. 1992) and is referred to as Paper II.

\section{Selection of objects}

In first instance, the objects selected are within 20 degrees of the galactic center. Three groups are distinguished. The first consists of nebulae for which the spectra were observed by us. The second are objects for which spectra have already been published elsewhere. The last group is formed by those nebulae for which the abundances have been available in the literature but for the sake of uniformity we have redone the analysis.

\subsection{Newly observed objects}

The sample of PNe studied by Gathier et al. (1983) forms the bulk of this group. These authors demonstrated that at least $90 \%$ are physically associated to the bulge region. The observations of this sub-sample ( $\sim 40$ objects) were carried out during several different sessions between 1982 and 1984 with the $3.6 \mathrm{~m}$ and $1.52 \mathrm{~m}$ telescopes at the European Southern Observatory (ESO), La Silla, Chile. The remaining 10 nebulae were observed in 1988 with the $1.9 \mathrm{~m}$ telescopes at the South African Astronomical Observatory (SAAO). This last set includes also some newly discovered PNe discussed by Pottasch et al. (1988), Ratag et al. (1990) and Kinman et al. (1988).

The ESO observations were made with the $3.60 \mathrm{~m}$ telescope (for the nebulae $1-4.2,2-2.4,2-3.3$ (VLE), $2-3.5$ (VLE), $2-4.1,2-6.1,3-4.3,7+1.2,353+6.2$, $354+4.1,357+7.1$ and $359-1.2$ (VLE) and with the $1.52 \mathrm{~m}$ telescope (for the rest of this group). Both telescopes were equipped with similar instruments: a Boller and Chivens spectrograph equipped with an IDS (Image Dissector Scanner) detector, and the same gratings were used in both cases. Spectra were obtained in two parts: blue spectra (range $3500-5380 \AA$ ) were observed at $114 \AA / \mathrm{mm}$, while red spectra $(4300-7400 \AA)$ were obtained at $171 \AA / \mathrm{mm}$, with a comfortable overlap between both spectra for consistency checks. Exposures consisted of succession of pairs of scans observed through two apertures (object and sky) separated by $60^{\prime \prime}$, the object being flipped from one aperture to the other between consecutive pairs of scans. The only difference between the two telescopes was the size of the aperture to cope with the different scales and yet achieve similar spectral resolutions. At the $3.60 \mathrm{~m}$, the apertures were $1^{\prime \prime} .8 \times 4^{\prime \prime}$ in size, yielding spectral resolutions (projected slit widths) of 5.3 and $8.0 \AA$ in the blue and in the red, respectively, while at the $1.52 \mathrm{~m}$ they were $4^{\prime \prime} \times 4^{\prime \prime}$ with corresponding resolutions of 5.0 and $7.5 \AA$. As a consequence, the $\mathrm{H} \beta$ fluxes obtained at the $3.60 \mathrm{~m}$ telescope are only lower limits, but they are close to total fluxes for point-like objects observed at the $1.52 \mathrm{~m}$.

The data reduction was performed in the usual way for IDS data, the two apertures being reduced independently to the end step of absolute fluxes and only averaged afterwards. Sky is subtracted from object observed in the same aperture, sequentially in time, but the integration times of a few minutes between successive flips are short enough to ensure that sky variations are negligible in normal observing conditions. Pixel to pixel sensitivity 
variations were removed by division by a normalised flat field, transformation to wavelength scale done with reference He-Ar spectra obtained before and after the exposures on the sky and conversion to absolute fluxes (with the shortcomings of small slits mentioned above) made through spectra of standard stars observed in exactly the same conditions. An initial correction to the raw data (elevation to the power 0.96 ), specific to these IDS, has been applied before any further reduction to take into account a probable non-linearity discovered by Rosa (1985). While some hesitation about the reality of the effect had persisted for some years, evidence is now conclusive about it and its correction. A similar effect is found for the Kitt Peak IITS (Intensified Image Dissector Scanner) (see Peimbert \& Torres-Peimbert 1987a) and has been corrected for in the data of Aller \& Keyes (1987) used below. But more important, the growing CCD data base now shows (for objects observed with both detectors) that the best agreement is obtained with corrected IDS data (see Leisy \& Dennefeld 1996, and references therein). One remaining question is the exact value of the [OIII] 5007 to 4959 line ratio, observed with all data sets to be close to $3.00( \pm 0.08)$ while the theoretical value is 2.88 .

The remaining objects $(2+1.1,3-4.7,3-4.3,3+3.1$, $4+6.2,5+4.1,357+4.1,359-2.4,359+3.4$ and $359+4.1$ ) were observed at SAAO with the $1.9 \mathrm{~m}$ telescope, equipped with the Cassegrain Grating Spectrograph and the Intensified Reticon Photon Counting System. Object and Sky were observed alternately through two entrance apertures of $1^{\prime \prime} .8 \times 6^{\prime \prime}$, separated by $30^{\prime \prime}$ on the sky. Two gratings were used: the first one, giving $210 \AA / \mathrm{mm}$ covered the full range of $3500-7600 \AA$ with $8 \AA$ resolution (FWHM) while the second one, yielding $100 \AA / \mathrm{mm}$, covered either the blue or the red range at $4 \AA$ resolution with the 1 .' 8 entrance slit. The observing strategy and the subsequent standard data reduction flow were similar to the one described above for the IDS data, therefore providing at the end an homogeneous set of data.

Based on the newly obtained spectra we also confirm the non-PN nature of some of the objects. The spectra of PK 3-4.6 (Ap1-1), PK 6+7.1 (M1 - 21), and PK 7+1.2 (M3-18) are typical of symbiotic stars as noticed by Acker et al. (1987). No emission line object was detected at the position of PK $0-02(\mathrm{~B} 13-22)$.

Considering the relatively greater uncertainty in the measurement of $\mathrm{H} \delta$, only the Balmer line ratios $\mathrm{H} \alpha / \mathrm{H} \beta$ and $\mathrm{H} \beta / \mathrm{H} \gamma$ were applied in the determination of interstellar distinction. The theoretical values for the decrement were taken from Brocklehurst (1971) for case B, $T_{\varepsilon}=$ $10^{4} \mathrm{~K}$ and $N_{\varepsilon}=10^{4} \mathrm{~cm}^{-3}$, except that for PK $0-2.3$ and PK 3-4.9 where the decrement for $T_{\varepsilon}=210^{4} \mathrm{~K}$ was used. The adopted $E(B-V)$ is obtained by averaging the results from $\mathrm{H} \alpha / \mathrm{H} \beta$ ratio and from $\mathrm{H} \beta / \mathrm{H} \gamma$, weighted in the ratio $3: 1$, to allow for the greater observational accuracy of the brighter Balmer lines (except for PK $355-2.4$ and PK $353+6.2$, where there are strong indications of self-absorption in Balmer lines). For PK $355-2.4$ and $\mathrm{PK} 353+6.2$, the $\log [F(\mathrm{H} \alpha) / F(\mathrm{H} \beta)]-\log [F(\mathrm{H} \beta)]$ diagram such as discussed by Osterbrock (1974; see also Barker 1978a, Fig. 1) has been used to estimate the optical depth of $\mathrm{H} \alpha$ due to Balmer line self-absorption as well as the "corrected" $E(B-V)$. The $\tau(\mathrm{H} \alpha)$ for both nebulae are between 10 and 15 . The resulting values of $E(B-V)$ derived from the Balmer decrement are given in Col. 7 of Table 2a. Another way of determining the extinction $E(B-V)$ is by comparing the radio continuum flux density with the $\mathrm{H} \beta$ flux. Assuming that the extinction is all external to the nebula, and by taking into account the wavelength dependence of the extinction, we can derive the $E(B-V)$, which is listed in Col. 8 of Table 2a. The values computed using both methods show, in general, a fair agreement, although in a small number of cases a large discrepancy is noticed. This discrepancy is largely caused by the uncertainty in the $\mathrm{H} \beta$ flux measurement. Many objects have been observed with a slit size smaller than the diameters of the nebulae, and it is certainly very difficult to estimate the fraction of the unobserved flux. This fraction depends on various factors such as the detail morphology of the nebula, the exact position on the nebular image where the slit has been centered on, and the surface distribution of the $\mathrm{H} \beta$ flux. In the cases of large discrepancy, we have always preferred the $E(B-V)$ derived from the Balmer decrement method. In other instances, the average values were generally adopted. These values, in combination with the reddening curve of Savage \& Mathis (1979) are then used to calculate the reddening-corrected intensities. These are given in Table 1 relative to $I(\mathrm{H} \beta)=100$.

\subsection{Objects with published spectra}

Recently Webster (1988) and Acker et al. (1989a) published the spectra of a large number of PNe. The first survey is intended for the bulge region, while the other is for the whole sky. Those objects have been selected which are not included in the groups described in Sects. 2.1 and 2.3. Webster has derived the abundances of $\mathrm{He}, \mathrm{O}$ and $\mathrm{N}$ in her sample by using the empirical ionization correction factors based on the similarities in ionization potential of some ions of different elements. In order to obtain a more homogeneous result we have reanalysed the spectra, using their published line intensities which are reddeningcorrected.

Acker et al. published the uncorrected intensities but provide also the logarithmic reddening corrections $c$ at $\mathrm{H} \beta$. As in the former sample, it is also possible to derive the extinction by comparing radio frequency and $\mathrm{H} \beta$ fluxes. The method used to derive the adopted $c$ values is similar to that described in Sect. 2.1. We have used these $c$-values together with the reddening curve mentioned in Sect. 2.1 to derive the unreddened intensities. We however note that, because of the survey nature of their project, the relatively short exposure times used by Acker et al. are 
generally not adequate to derive high quality abundances from their data which furthermore, were not corrected for the IDS linearity problem. In addition, the important [O II] 3727 line was not observed. For the discussion on the uncertainties in line intensity measurements, the reader is referred to the original papers.

\subsection{Objects with published abundances}

Objects in this category are taken from the work of Aller \& Keyes (1987). They are those nebulae not included in the first group. As mentioned earlier, a similar algorithm to that employed in this work had been used to derive the abundances. It should also be noted that temperatures and densities derived from our data were used by Aller \& Keyes (1987) for some objects in common, due to the uncertainties resulting from the low elevation observations at Lick Observatory. Therefore only objects not observed by us were retained from their work.

Further selection has subsequently lead to exclude those nebulae which are likely to be foreground objects on the basis of luminosity (greater than $210^{4} L_{\odot}$ if placed at the GC) size (greater than $20^{\prime \prime}$ in diameter), and the available information on the interstellar extinction along the line of sight. Objects rejected from the sample are NGC 6369, M1 - 26, Hb5, M1 - 40, Tc 1, Hb4, Hb6, NGC 6567, NGC 6578 and NGC 6629.

In total about 110 nebulae are included in the final sample. This is around $85 \%$ of the original sample. Although it is still possible that a very few foreground objects remain, the fraction is certainly much less than $10 \%$. The number of PNe as a function of angular distance from the center is shown as a histogram in Fig. 1. They are mostly within 10 degrees of the Galactic Center. Selection effect, due to the very large extinction, probably explains the lack of objects within 4 degrees of the center.

\section{Interpretation of the spectra/analysis of data}

\subsection{Excitation class}

In most cases, even by looking at a reddened spectrum, one can roughly assign the excitation level of the emitting nebula. The classification scheme applied here is the one suggested by Aller (1956) with a slight modification. A scale from 0 to 10 is used in the system. For low excitation (classes $\leq 5)$, the $[\mathrm{O}$ II $] \lambda 3727 /[\mathrm{O}$ III $] \lambda 4959$ ratio is used as a main indicator, while for the higher classes, the ratio of He II $\lambda 4686$ to He I $\lambda 5876$ is an imporant criterion. In many cases, the ratio of lines originating from $[\mathrm{Ar}$ III], [Ar IV] and [Ar V] have been taken into consideration (see the discussion by Ratag \& Pottasch 1990). The limited sensitivity and wavelength coverage in the spectra analyzed in this program do not allow us to use the more sensitive indicators such as the ratio $[\mathrm{Ne} \mathrm{V}] \lambda 3425 /[\mathrm{Ne}$ III $] \lambda 3868$ in the

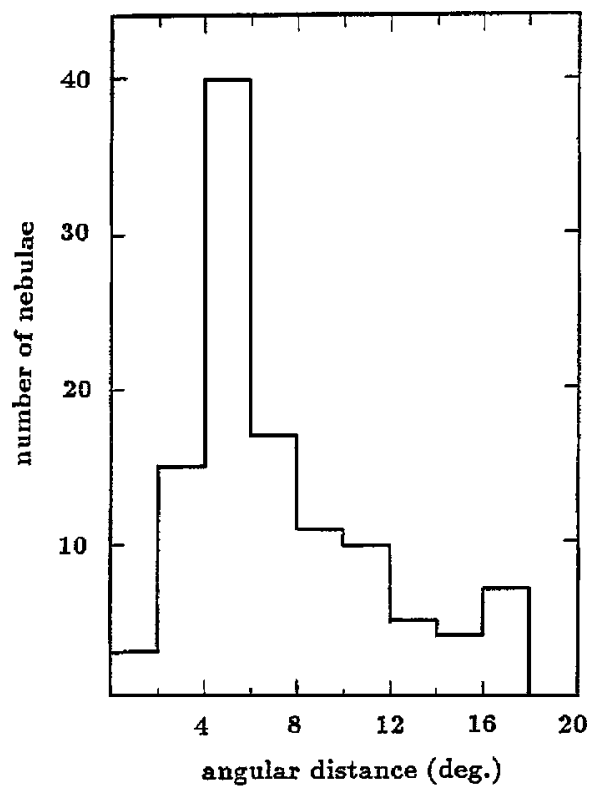

Fig. 1. Histogram of the angular distance from the Galactic center of the bulge PNe studied in this program

nebulae with very high excitation. The classification beyond class 7 should be thus considered as uncertain by about 1 class.

The most important parameters which contribute to determine the excitation class of a nebula are the effective temperature of the exciting star, the geometrical dilution factor, the optical depth in the Lyman continuum, and the abundances. In order to eliminate the dependence on the last factor, we should, in principle, avoid using a ratio between lines originating from different elements, viz. the ratios $[\mathrm{O}$ III $](\lambda 4959+5007) / \mathrm{H} \beta$, He II $\lambda 3868 / \mathrm{H} \beta$, in determining the class as the abundances can vary by a large factor from nebula to nebula. An example of a system which neglects the abundance variational effect is the one proposed by Feast (1968), and used by Webster (1988) in classifying the nebulae in her sample.

The excitation class distribution of the bulge PNe considered here is shown in Fig. 2. The distribution has a peak around the classes 5 and 6 . Comparisons are made with the bright nearby nebulae and the (smaller) bulge sample studied by Webster (1988). The former are taken from Aller \& Czyzak (1983), Aller \& Keyes (1987), Pottasch (1984), Peimbert et al. (1987b). The nearby nebulae seem to show an excess of high excitation objects and lack of low excitation nebulae compared to the total bulge sample. The bulge distribution is shifted toward the lower excitation by about 1.5 to 2 classes with respect to the nearby sample. Some possibilities can be put forward to explain this. The difference in the high excitation range could be due to selection effect, as the PNe with high central star temperatures tend to have a relatively larger size and lower surface brightness, and thus are difficult to 
observe in the bulge region. Although this could explain the high excitation excess, it is certainly not able to explain the lack of objects in the opposite extreme. The low excitation nebulae must be much easier to observe if they are nearby. Another alternative is that the bulge PNe are ionized by stars which have on average lower effective temperatures. Ratag et al. (1990) examined this tendency and argued that it is independent of the selection effect just mentioned above. They found the mean $T_{\text {eff }}^{*}$ for the bulge $\mathrm{PNe}$ of about $45000 \mathrm{~K}$, while for the non-bulge sample, independent of size, the value is almost twice as high. We conclude that the difference in the excitation class distribution between the bulge sample and the nearby nebulae is real and simply reflects the difference in their $T_{\text {eff }}^{*}$ distribution.

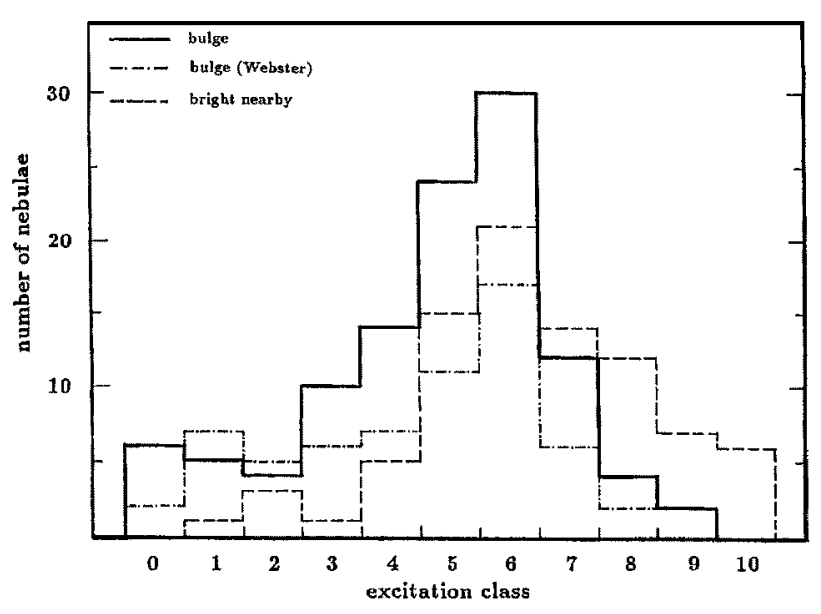

Fig. 2. The excitation class distribution of the bulge PNe studied in this work (full thick) is compared with that of the nearby nebulae (dashed) and that of the smaller bulge PN sample studied by Webster (1988) (dashed-dotted). The last sample shows a similarity to the total bulge sample. Both are shifted by approximately 1.5 to 2 classes toward lower excitation range with respect to the nearby nebulae. This is probably due to the difference in their cental star effective temperature distribution

The second comparison is made with Webster's sample. Using the Feast (1968) system she found that the excitation class distribution is fairly uniform over the whole excitation range. As pointed out previously, the Feast system is affected by the abundance. We have reclassified the objects according to Aller's scheme and display the distribution in Fig. 2 as a dashed-dotted histogram. We include also a few objects, mainly the low excitation one, which do not have the important lines necessary for the plasma diagnostics and therefore have been excluded from the total bulge sample. The resulting distribution clearly shows a non-unformity, and is very similar to the total sample, having a peak at about classes 5 and 6 .

\subsection{Plasma diagnostics: Electron temperatures and densities}

The plasma diagnostics were done in $T_{\varepsilon}-N_{\varepsilon}$ diagrams where we plotted the dependence on temperature and density of the available forbidden line intensity ratios. In all computations we made use of the atomic parameters compiled by Mendoza (1983) supplemented with new data recommended by Clegg (1989). We calculated the populations of up to fifteen levels of each ion in order to obtain the theoretical intensity ratios.

The electron temperature $T_{\varepsilon}$ indicators in our spectra are the ratios of $[\mathrm{O} \mathrm{III}](\lambda 4959+\lambda 5007) / \lambda 4363$ and $[\mathrm{N} \mathrm{II}](\lambda 6548+\lambda 6583) / \lambda$ 5755. On several occasions the ratios $[\mathrm{O}$ II $] \lambda 7325 / \lambda 3727$ and $[\mathrm{S} \mathrm{II}](\lambda$ 4068+ $\lambda$ 4076)/ $(\lambda 6717+\lambda 6731)$ provide secondary information. The uncertainties in $T_{\varepsilon}$ determinations are mainly due to the weakness of $\lambda 4363$ and $\lambda 5755$ lines. For a relatively faint $\lambda 4363$ line, an uncertainty in intensity of about $25 \%$ should be allowed. This leads to a resulting $T_{\varepsilon}$ with an imprecision of about $10 \%$. A relatively strong $\lambda 4363$ line, with an accuracy of better than $15 \%$, would lead to a $T_{\varepsilon}$ with uncertainty of about $5 \%$. The $[\mathrm{N}$ II $] \lambda 5755$ lines are generally less precise, and we suggest that the accuracy in the resulting $T_{\varepsilon}[\mathrm{N}$ II $]$ is between $5 \%$ to $15 \%$ worse than that related to the $T_{\varepsilon}[\mathrm{O} \mathrm{III}]$.

The main density indicator used in this program is the [S II] doublet at about $6725 \AA$. Reasonably good atomic data for [Ar IV] are now available thanks to the computation by Zeippen et al. (1987). The line intensity ratio [Ar IV] $\lambda 4711 / \lambda 4740$ can be used as a reliable diagnostic for electron density. The use of this line ratio usually involves a self-consistent iterative procedure in order to subtract the possible contribution of $\mathrm{He}$ I and [Ne IV] emission at $\lambda$ 4711. The He I can be easily predicted from the He I $\lambda 5876$, while the subtraction of the [Ne IV] lines requires us to examine the ionization structure to estimate the ratio of $N\left(\mathrm{Ne}^{3+}\right)$ to $N\left(\mathrm{Ne}^{++}\right)$. The latter is usually well represented in the spectra at $\lambda 3868$ and $\lambda 3968$ (blended with $\mathrm{H} \varepsilon$ ). Having subtracted the contributions of $\mathrm{He}$ I and [Ne IV] lines we redetermined the $N_{\varepsilon}$ with the corrected [Ar IV $] \lambda$ 4711, and eventually remodelled the nebula. The procedure was repeated (for 4 to 5 iterations) until a satisfactory consistency was achieved. In a small number of cases, the density sensitive doublets of [C III] are available and have also been applied. The advantage of using [Ar IV] and $[\mathrm{C}$ III $]$ line ratios is that for the medium to high excitation and for the high density $\left(N_{\varepsilon} \geq 5000 \mathrm{~cm}^{-3}\right)$ nebulae the derived electron density is more representative for the whole nebula than that derived from the [S II] doublet. Unfortunately, the corresponding lines are usually much weaker than those coming from the $\mathrm{S}^{+}$ions.

Since the distance to the nebulae are reasonably well known ( $d=7.7 \mathrm{kpc}$; Reid 1989) with an uncertainty of probably less than $20 \%$, and because the sizes, the radio continuum flux densities and/or the $\mathrm{H} \beta$ flux 
measurements are already available for almost all the nebulae, it is possible to determing the $N_{\varepsilon}(\mathrm{rms})\left(=N_{\varepsilon} \epsilon^{1 / 2} ; \epsilon\right.$ is the filling factor defined as the ratio between the filled and the total volume) with reasonably good accuracy. To calculate the $N_{\varepsilon}$ (rms), the equation

$N_{\varepsilon} \epsilon^{1 / 2}=4.9610^{2} \frac{S_{6 \mathrm{~cm}}^{1 / 2} T_{\varepsilon}^{1 / 4}}{\theta^{3 / 2} d^{1 / 2} t^{1 / 2}}$

(Spitzer 1978) was used. Here $S_{6 \mathrm{~cm}}$ is expressed in $\mathrm{mJy}$, $T_{\varepsilon}$ in $\mathrm{K}, d$ in $\mathrm{kpc}$, and $\theta$, the angular radius of the source, in arcsec, and $t \equiv 1+[2 x y /(1+y+x y)]$, where $x=$ $N\left(\mathrm{He}^{+} / N\left(\mathrm{H}^{+}\right)\right.$and $y=N(\mathrm{He}) / N(\mathrm{H})$.

The electron densities derived from the [S II] lines are plotted against the $N_{\varepsilon}(\mathrm{rms})$ in Fig. 3 . In this figure we distinguish the small size objects (with a diameter $\leq 3$ arcsecs) from the larger ones. Some important remarks can be made from the figure. The spread is usually interpreted as due to the non-uniformity in the densities. Since for most of bulge nebulae the observed lines come from the whole object rather than from a small region, the filling factor $\epsilon$ can be estimated such that $N_{\varepsilon}(\mathrm{FL}) \epsilon^{1 / 2}=N_{\varepsilon}(\mathrm{rms})$. For the nebulae lying below the line corresponding to $\epsilon=1.0$, this brings a special problem since the derived $\epsilon$ will be larger than unity. This is generally not caused by the error in the adopted distance or in the size determination. Even if the distances are wrong by about $2 \mathrm{kpc}$, the discrepancies from the line with $\epsilon=1.0$ are still large. Allowing an error of about $25 \%$ in the size determinations can shift the points by only about 0.145 dex to the left. The deviations are still present.

Another further point is of interest regarding Fig. 3. The problem we just mentioned is almost always met when the $N_{\varepsilon}(\mathrm{rms})$ is higher than about $5000 \mathrm{~cm}^{-3}$, and when the angular diameter is less than about 3 arcsecs. A higher density, and thus a higher optical depth, will result in a larger drop in the specific intensity $J(v)$ as we proceed away from the star. If the distance from the ionizing star to the nebula is still relatively small, the dilution factor for the inner region can differ by a large factor from that for the outer region. As the nebula evolves, expanding with an assumed uniform velocity, this difference will become smaller. Consequently, in the case of small, dense and especially medium to high excitation nebulae, the electron density obtained from the $[\mathrm{SII}]$ lines is likely to represent the region near the periphery where the ionization has dropped by a large factor and thus having lower $N_{\varepsilon}$. The best photoionization models of these nebulae show that this is indeed the case. Accordingly, for such nebulae we have always adopted the $N_{\varepsilon}$ (rms) as the relevant density to derive the atomic hydrogen number density $N_{\mathrm{H}}$. For this particular reason we have reanalysed two objects, PK $357+2.4$ and PK $356-4.1$, from Aller \& Keyes (1987) sample. In their analysis, electron densities of respectively, $7500 \mathrm{~cm}^{-3}$ and $4000 \mathrm{~cm}^{-3}$ were adopted, while the radio continuum measurements (Gathier et al. 1983; Zijlstra et al. 1989) indicate that values much higher than
$10^{4} \mathrm{~cm}^{-3}$ should be used. In the case of PK $356-4.1$ the newly derived abundances differ only by about $15 \%$ to $25 \%$ from the previous results but for PK $357+2.4$ these differences are, on average, $40 \%$ which stress the importance of the problem.

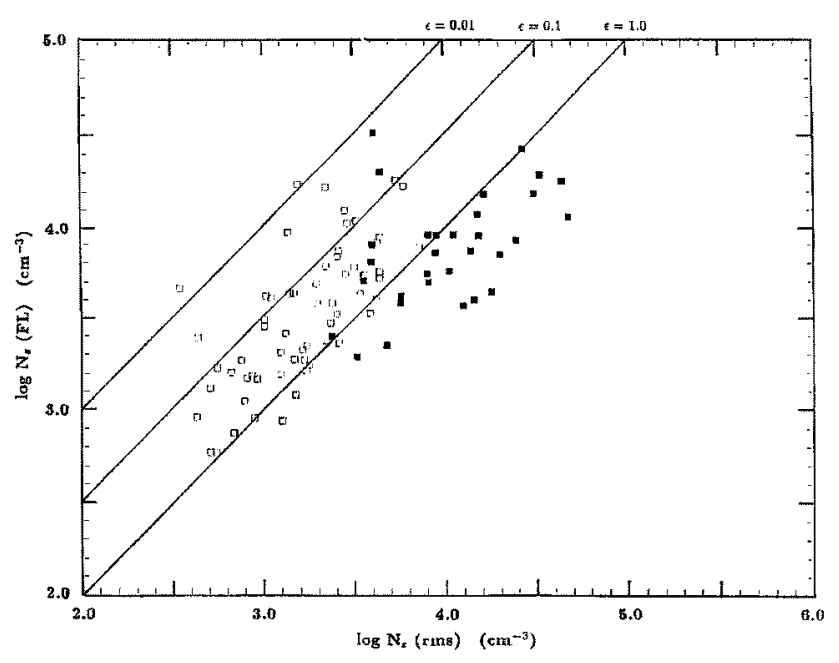

Fig. 3. The electron density derived from the radio continuum measurements, $N_{\varepsilon}(\mathrm{rms})=N_{\varepsilon} \epsilon^{1 / 2}$ ( $\epsilon$ is the filling factor) plotted against the density obtained from the forbidden line ratio of [SII]. The three straight lines are the expectations of a simple model with filling factor $\epsilon=0.01, \epsilon=0.1$, and $\epsilon=1.0$ (uniform). The nebulae with a diameter equal to or less than 3 arcsecs are represented by the filled symbol

Tables $2 \mathrm{a}$ and $2 \mathrm{~b}$ list some important parameters of the objects investigated in the present program. Table $2 \mathrm{a}$ is for the newly observed objects discussed in Sect. 2.1, and Table $2 \mathrm{~b}$ is for those of which the spectra are taken from literature (Sect. 2.2). Both tables are arranged as follows. In Cols. 1 and 2, we give the PK-designation and the usual name of the nebula. Column 3 lists the excitation class as discussed in Sect. 3.1. The radio continuum flux density at $6-\mathrm{cm}$ is given in Col. 4 in units of mJy. They are mostly from the works of Gathier et al. (1983) and Zijlstra et al. (1989). The $\mathrm{H} \beta$ flux (in units of erg $\mathrm{cm}^{-2}$ $\mathrm{s}^{-1}$ ) is tabulated in Col. 5 in logarithmic form. The next Col. 6 gives the angular diameter of the nebula in arcsecs, measured mostly by the VLA by Gathier et al. and Zijlstra et al. In a few cases the optical diameters were adopted. The $E(B-V)$ s derived by using the Balmer decrement method and by comparing the expected $\mathrm{H} \beta$ flux, based on radio continuum measurements, with that optically observed are given respectively in Cols. 7 and 8 . The electron temperature obtained from the plasma diagnostics are listed in Cols. 9 (O III), 10 (N II) and 11. The $T_{\varepsilon}$ presented in Col. 11 is the average expected for the whole nebula. In the next Col. (12), we list the electron density $N_{\varepsilon}$ as derived from the forbidden line intensity ratios ([S II $]$, [Ar IV] and [ClIII]). These various line 
ratios allow a density diagnostic up to about $20000 \mathrm{~cm}^{-3}$. Column 13 gives $V_{\mathrm{LSR}}$, the radial velocity in $\mathrm{km} \mathrm{s}^{-1}$ referred to the local standard of rest as adopted from the catalog of Schneider et al. (1983). For Table 2a there is an additional column, i.e. Col. 14 , which gives the ratio of the observed continuum flux at $\lambda 5325 \AA$ to $\mathrm{H} \beta$ flux density, appropriate for the derivation of the central star effective temperature. The total infrared flux $F_{\text {tot }}^{\mathrm{IR}}$, based on the IRAS measurements and derived by integrating between $10 \mu \mathrm{m}$ and $100 \mu \mathrm{m}$ are shown in the 15 th column of Table $2 \mathrm{a}$ and $14 \mathrm{th}$ of Table $2 \mathrm{~b}$. In Col. 16 of Table $2 \mathrm{a}$ and Col. 15 of Table $2 \mathrm{~b}$, we list the infrared excess (IRE) computed by using equation (VIII-11) of Pottasch (1984), taking into account the dependence on density and in a small number of cases, on the optical depth at 6 - $\mathrm{cm}$. The letter symbols given in the last column are references listed at the end of the tables.

\section{Abundance derivation}

An uncertainty in deriving abundances from analysis of spectral line intensities of planetary nebulae has been the distribution of atoms among unobserved ionization stages. Two basic methods had been introduced: (a) employing ad hoc empirical expressions based on simple proportionalities of ionization potentials; (b) theoretical models constructed to reproduce the observed line intensities. The first method has been employed since the pioneering studies of Bowen \& Wyse (1939) and of Wyse (1942). Refinements have been made by numerous investigators (Seaton 1968; Peimbert \& Costero 1969; Peimbert \& Torres-Peimbert 1971; Barker 1978b, 1980; Natta et al. 1980). The ionization correction factors (ICFs) are established on the ground of ionization potential similarities among some ions of different elements, or empirically formulated following several tests in some well-observed nebulae.

The second method, employing theoretical models, is known to be very difficult because it is model dependent. Furthermore, it requires a complicated radiative transfer treatment, as compared to the on-the-spot approximation (See Harrington et al. 1982). Among the limitations corresponding to the use of theoretical models are the difficulties in treating the density fluctuations, the lack of satisfactory models for the ionizing stars (especially those with $T_{\text {eff }}^{*} \geq 85000 \mathrm{~K}$ ), and the unrealistic assumptions about nebular morphology.

Our analysis occupies a middle ground between those two above-mentioned methods. The procedure is primarily due to Shields et al. (1981) and has been extensively used by Aller \& Czyzak (1983) and Aller \& Keyes (1987). Here, the theoretical models are employed as interpolation devices to derive ICFs, which are eventually applied to ionic concentrations, $N\left(\mathrm{X}^{+i}\right) / N\left(\mathrm{H}^{+}\right)$, to obtain the total abundances. The comparison of the model abundances and those computed with the help of model ICFs and ionic concentrations is chosen as the main criterion for the judgement of the process.

More complete discussions on the nebular modelling, together with the background physics, have been presented by various authors (Aller 1984; Yorke 1988; Keyes \& Aller 1978). We will not attempt to repeat them in this paper. Only a brief outline procedure of the modelling and some very important aspects involved in it will be described here.

The theoretical model is originally due to Balick (1975), but is improved to accommodate differences in nebular geometry as well as to take into account additional ions, important physical processes such as dielectronic recombination and charge exchange, and several revised atomic parameters such as charge-exchange cross-sections, collision strengths, absorption coefficients, recombination coefficients and $A$-values. For the selection of the adopted atomic parameter we refer to the papers by Shields et al. (1981), Mendoza (1983) and Clegg (1989). At the outset, these parameters together with the equations for various physical processes, radiative transfer, etc. are stored in the code. The treatment of the radiative transfer is simplified by the on-the-spot approximation. In constructing the model that will be appropriate as an interpolation device, we firstly tried to reproduce certain line intensity ratios indicating the level of excitation such as He II $\lambda 4686 / \mathrm{He} \mathrm{I} \lambda$ 5876 , [O III] $(\lambda 4959+\lambda 5007 / \lambda 4363$, and in some cases, $[$ Ar III $] \lambda 7135 /[$ Ar IV $] \lambda$ 4740. The last is used instead of the ratio $[\mathrm{Ne} \mathrm{V}] \lambda 3425 /[\mathrm{Ne}$ III] $] \lambda 3868$, which cannot be extracted from the available spectra. In the second step, we attempted to represent the observed unreddended line intensities as accurately as possible. In performing those efforts we treated the radius $R_{*}$ of the central star, the nebular truncation radius, the surface flux distribution $\pi F_{\mathrm{v}}^{*}$ from the central star, the hydrogen number density $N_{\mathrm{H}}$, and the nebular chemical composition as adjustable parameters. Modification of the first parameter turned out to have minor effects on the degree and pattern of excitation and negligible contributions on the finally derived abundances. Initial abundances for the observed elements are established by using the abundances computed employing the ad hoc formulae (see the discussion earlier in this section). Unobserved elements such as $\mathrm{Na}, \mathrm{K}, \mathrm{Ca}, \mathrm{Mg}$, $\mathrm{Si}$, etc. were handled as having ratios to oxygen similar to the corresponding solar values. For a large sample this is probably justified. In spite of the fact that it might not be true in individual cases, for the present purpose the assumption seems to be sufficient enough. In fact, trials by varying the ratios up to a factor of 3 showed very little effect on the overall ionization structure, and thus produced ICFs very similar to those produced by the model constructed by adopting solar ratios.

In the case of carbon we use a different trick. This element, as well as $\mathrm{He}, \mathrm{N}$, and $\mathrm{O}$, is likely to be affected by the CNO tri-cycle, and probably also by the triple$\alpha$ process, which occurred during the previous stages of 
evolution of the central star. As has been shown by observational results, there is no simple correlation between the abundance ratio of $\mathrm{C}$ (with respect to either $\mathrm{H}$ or $\mathrm{O}$ ) and the related abundance or abundance ratios involving $\mathrm{He}, \mathrm{H}, \mathrm{N}$ and $\mathrm{O}$ (Zuckermann \& Aller 1986). In the sample of $\mathrm{PNe}$ studied by them, variations in $\mathrm{C} / \mathrm{O}$ and $\mathrm{N} / \mathrm{O}$ ratios by factors of respectively 58 and 100 are noticed. The ratios of the total abundance of $\mathrm{C}, \mathrm{N}$ and $\mathrm{O}$ denoted by " $\Sigma$ ", relative to the related value in the solar system $\left(\Sigma_{\odot}\right)$, however, show much smaller variation, since the CNO tri-cycle probably simply permuted those three elements. From about 40 objects, a mean $\Sigma / \Sigma_{\odot}$ of 0.99 with a standard deviation $\sigma$ of 0.43 (less than a factor of 3 ) is obtained. This mean value has been adopted to estimate the carbon abundance throughout our sample. Similarly to that in the cases of other unobserved elements discussed previously, tests by varying the $\Sigma / \Sigma_{\odot}$ have also been made. The test results showed that variation up to a factor of 3 would usually lead to discrepancies in total abundances which would become incompatible with observed intensities. But obviously nothing can replace direct observation of carbon lines.

In the following, let us consider the examples of the procedure as applied to two cases. They are among the best observed. The first nebula $(\mathrm{PK} 0+17.1)$ is representative of the low to medium excitation nebulae, while the second (PK 359-2.3) is typical of the medium to high excitation.

\section{1. $P K 0+17.1$ (Excitation class 3.5)}

From the plasma diagnostics we obtained $N_{\varepsilon}=$ $9150 \mathrm{~cm}^{-3}$. For the nebular model we adopt $N_{\mathrm{H}}=$ $8500 \mathrm{~cm}^{-3}$, which gives $\left\langle N_{\varepsilon}\right\rangle=9200 \mathrm{~cm}^{-3}$ for the emitting volume. For the stellar energy distribution we used Clegg \& Middlemass' (1987) non-LTE model atmospheres extrapolated for $T_{\text {eff }}^{*}=37500 \mathrm{~K}, \log g=4.5$. Based on the ionization structure of hydrogen, with neutral hydrogen in the outer part, this nebula is apparently ionization bounded. In about $25 \%$ to $35 \%$ of the ionized region, helium is still in neutral form. The ionization structures of the best model are shown in Figs. 4b and 4c. Figure 4a displays the $T_{\varepsilon}$ distribution as fixed by the energy-balance. Throughout the inner region of the nebula up to a radius of about $60 \%$ of the Strömgren radius, the $T_{\varepsilon}$ lies between $8200 \mathrm{~K}$ and $8450 \mathrm{~K}$. It is approximately the region where the [O III] lines come from. The mean predicted $T_{\varepsilon}$ (O III) of $8350 \mathrm{~K}$ fits very well the observed one. In the outer region the electron temperature increases and has a peak of $9250 \mathrm{~K}$ at about $r=0.029 \mathrm{pc}$. This rapid rise may not be due to the lack of coolants near the outer boundary but rather a consequence of the more important effect of the hardening of the radiation field (Aller et al. 1980; Aller 1984). The average temperature in this outer region agrees very well with the observed $T_{\varepsilon}(\mathrm{N}$ II $)$.
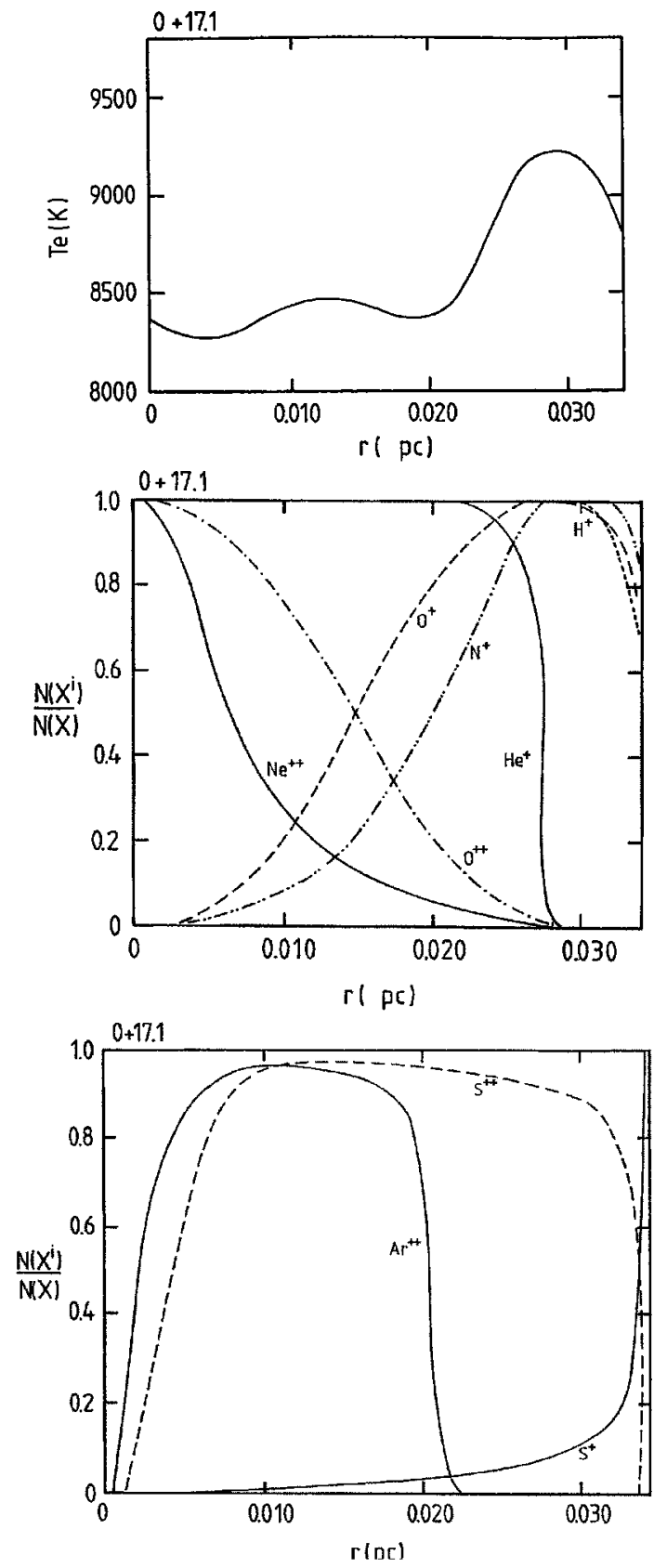

Fig. 4. The temperature a) and the ionization $\mathbf{b}, \mathbf{c})$ structures of the best model for PK 0+17.1 (excitation class 3.5). Notice that the ionization of helium has dropped to zero at radius $\tau$ of approximately $0.028 \mathrm{pc}$

\subsection{PK 359-2.3 (Excitation class 6)}

The electron temperatures derived from the plasma diagnostics of this nebula gives the values of $9300 \mathrm{~K}$ and $13100 \mathrm{~K}$ for respectively, $T_{\varepsilon}(\mathrm{O}$ III $)$ and $T_{\varepsilon}(\mathrm{N}$ II $)$. The electron density obtained from the [S II] doublet is $4430 \mathrm{~cm}^{-3}$. This is much smaller than the rms-density of $17900 \mathrm{~cm}^{-3}$ derived from the radio continuum meansurement at $6 \mathrm{~cm}$ 
(Gathier et al. 1983). As argued in Sect. 3.2, the last value should be used as representative of the majority of the ionized region, and a filling factor $\epsilon$ of unity should be appropriate for the model. The ionization structure of this nebula confirms that the [S II] lines are likely to originate from the outer regions. For the whole region, we adopt $N_{\mathrm{H}}=15900 \mathrm{~cm}^{-3}$, yielding $\left\langle N_{\varepsilon}\right\rangle=17800 \mathrm{~cm}^{-3}$ for the emitting volume. The central star surface flux distribution with $T_{\text {eff }}^{*}=80000 \mathrm{~K}$ and $\log g=4.70$, interpolated from the models published by Clegg \& Middlemass (1987), is found to produce a reasonably good nebular model for PK 359-2.3. The electron temperature and the ionization structure of some important ions are shown in Figs. 5a, b and c. The $T_{\varepsilon}\left(\mathrm{O}\right.$ III) and $T_{\varepsilon}(\mathrm{N}$ II $)$ are both nicely fitted by the model. For the same reason as in the former case, the nebula is likely to be radiation bounded. As noticed in the previous case, there is an increase of temperature near the outer boundary due to the hardening of the radiation field. It should be noted that in both cases considered here the electron density is far above the average. For lower density $\mathrm{PNe}\left(N_{\varepsilon} \leq 3000 \mathrm{~cm}^{-3}\right)$, the typical cases of $T_{\varepsilon}$ distribution roughly follow the scenario put forward by Kaler (1986). In PK 359-2.3, $\mathrm{He}^{++}$just appears in the region close to the ionizing star. We expect the presence of oxygen in the stages higher than $\mathrm{O}^{+2}$ in that region. The greatest difficulty in this case is when we are dealing with nitrogen. The ion $\mathrm{N}^{+}$, which is the only representative of nitrogen in the spectra, appears only in the very small region near the periphery. The ICF for this element is, consequently, very large and uncertain. The close agreement of the ICF and model results found in PK 359-2.3 shows however the problem to be less severe than expected. It could become more important for very high excitation objects.

In Table 3 we compare the predicted and the observed line intensities for the two cases above. As occurred also in most cases, although the overall agreement is reasonably fair, large discordances were often encountered when dealing with lines originated from ions involving $p^{3}$ configuration (e.g. [Ar IV], [S II], [Cl III], [O II]). Some of the difficulties may be caused by effects of dense partially ionized blobs and condensations, which are not handled in the theoretical models and which may be indeed present, and some may arise from imprecisions in atomic parameters.

The temperature and ionization structures are then employed as a guide in deriving the ionic concentrations and the ICFs shown, respectively, in Cols. 3 and 5 of Table 4a (PK 0+17.1) and Table 4b (PK359-2.3). The ionic abundances $N\left(\mathrm{X}^{+i}\right) / N\left(\mathrm{H}^{+}\right)$for each observed line intensities (Col. 2) as corrected for interstellar extinction and the relevant $T_{\varepsilon}$ and $N_{\varepsilon}$, using a code which solves the equations of statistical equilibrium. In the computation concerning helium, the collisional effects in He I lines have been taken into account. The correction for that has been done following the results of Clegg (1987). It has an average effect of lowering the helium abundance. Column 4
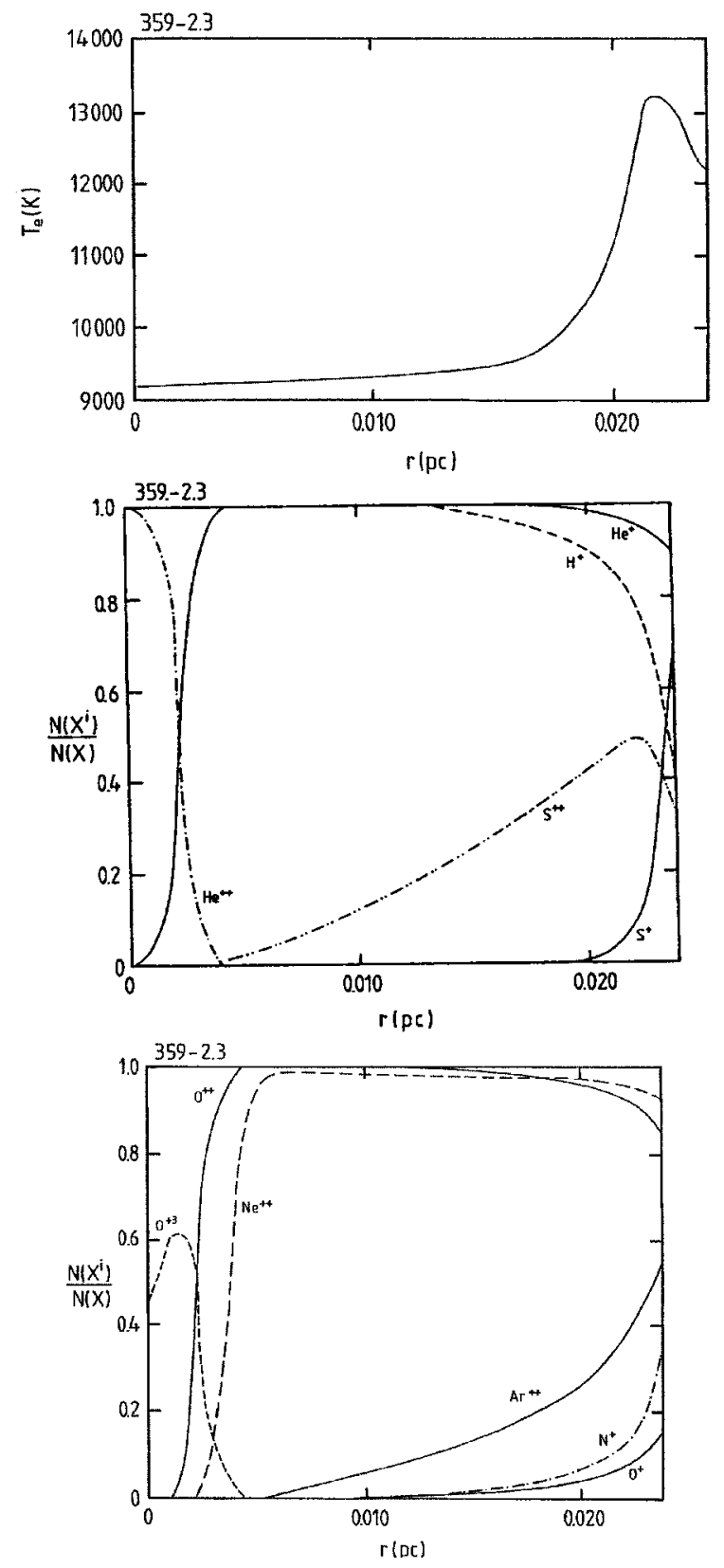

Fig. 5. The temperature a) and the ionization b, c) structures of the best model for PK 359-2.3 (excitation class 6). A rapid rise in the electron temperature near the outer boundary, as in the case of PK $0+17.1$, is apparently due to the hardening of the radiation field as hydrogen becomes neutral (see text). $\mathrm{He}^{++}$has just appeared in the very inner region, close to the illuminating star. Notice that the $\mathrm{S}^{+}$ions are concentrated in the region where the ionization of hydrogen has dropped by a large factor 
Table 3. Representation of line intensities in PK $0+127.1$ and PK $359-2.3$

\begin{tabular}{|c|c|c|c|c|c|}
\hline \multirow{2}{*}{$\lambda(\hat{A})$} & \multirow{2}{*}{$\begin{array}{l}\text { Identifi- } \\
\text { cation }\end{array}$} & \multicolumn{2}{|c|}{ PK $0+17.1$} & \multicolumn{2}{|c|}{ PK $350-2.3$} \\
\hline & & I(obs.) & $I$ (pred.) & I(obs.) & $\mathrm{I}$ (pred.) \\
\hline 5876 & HeI & 12.7 & 12.2 & 15.5 & 15.0 \\
\hline 4471 & HeI & 4.20 & 4,39 & 6.50 & 6.81 \\
\hline 4886 & HeII & 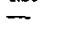 & $\rightarrow$ & 17.1 & 17.8 \\
\hline 5200 & {$[\mathrm{NI}]$} & 0.58 & 0.62 & - & - \\
\hline 6583 & [NII] & 99.0 & 101 & 61.4 & 60.2 \\
\hline 5755 & [NI] & 1.50 & 1.80 & 1.92 & 1.80 \\
\hline 6300 & [On] & 2.20 & 2.01 & 一 & 一 \\
\hline 3727 & [OII] & 163 & 180 & 32.4 & 34.7 \\
\hline 7325 & fom & 25.0 & 28.0 & 9.20 & 9.02 \\
\hline $4959+5007$ & IOIIII & 390 & 400 & $\begin{array}{l}1.20 \\
1222\end{array}$ & 1250 \\
\hline $\begin{array}{l}4909+0001 \\
4363\end{array}$ & [OIII] & 1.10 & 1.25 & 4.60 & 4.71 \\
\hline 3868 & [NeIII] & 11.0 & 12.1 & 79.1 & 83.3 \\
\hline 5517 & ClIm & 0.24 & 0.20 & - & 一 \\
\hline 5537 & [CIII] & 0.33 & 0.27 & - & 一 \\
\hline 6717 & [SII] & 2.70 & 2.89 & 0.99 & 0.96 \\
\hline & [su] & 5.00 & 5.30 & 1.71 & 1.75 \\
\hline 6312 & [sIII] & 1.00 & 1.05 & 1.70 & 1.64 \\
\hline 7135 & {$\left[A_{T}[\mathrm{II}]\right.$} & 7.70 & 8.88 & 13.6 & 14.9 \\
\hline 4740 & 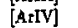 & $0.15:$ & 0.2 & 4.45 & 4.60 \\
\hline
\end{tabular}

lists the summation of all observed ionization stages. The fraction of this summation is equal to $1 / \mathrm{ICF}$. The values in Cols. 4 and 5 are then used to derive the abundances in Col. 6. The last column lists the abundances obtained from the best mode. In the next section we will discuss the comparisons of those two abundances.

Table 4. a) Analysis of PK $0+17.1$

\begin{tabular}{|c|c|c|c|c|c|c|}
\hline \multirow{2}{*}{ Ion } & \multirow{2}{*}{$\lambda(\dot{A})$} & \multirow{2}{*}{$\frac{N\left(X^{+i}\right)}{N\left(H^{+}\right)}$} & \multirow{2}{*}{$\Sigma \frac{N\left(X^{+i}\right)}{N\left(H^{+}\right)}$} & \multirow{2}{*}{ ICF } & \multicolumn{2}{|c|}{$N(X) / N(H)$} \\
\hline & & & & & ICF method & Model \\
\hline $\mathrm{He}^{+}$ & 5876,4471 & 0.0841 & 0.0841 & 1.29 & 0.109 & 0.104 \\
\hline $\mathrm{N}^{+}$ & 6583,6548 & $2.70 \times 10^{-8}$ & $2.70 \times 10^{-5}$ & 2.68 & $7.24 \times 10^{-5}$ & $6.79 \times 10^{-5}$ \\
\hline $0^{+}$ & 3727,7325 & $2.70 \times 10^{-4}$ & $4.90 \times 10^{-4}$ & 2.01 & $4.83 \times 10^{-4}$ & $5.00 \times 10^{-4}$ \\
\hline$O^{++}$ & 4959,5007 & $2.20 \times 10^{-4}$ & & & & \\
\hline $\mathrm{Ne}^{++}$ & 3868 & $2.10 \times 10^{-5}$ & $2.10 \times 10^{-5}$ & 2.26 & $4.78 \times 10^{-8}$ & $4.95 \times 10^{-5}$ \\
\hline$s^{+}$ & 6717,6731 & $6.30 \times 10^{-7}$ & $4.73 \times 10^{-6}$ & 1.71 & $8.13 \times 10^{-6}$ & $7.80 \times 10^{-6}$ \\
\hline $\mathrm{Cl}^{++}$ & 5517,5537 & $2.20 \times 10^{-7}$ & $2.20 \times 10^{-7}$ & 1.86 & $4.11 \times 10^{-7}$ & $3.80 \times 10^{-7}$ \\
\hline $\begin{array}{l}A r^{++} \\
A r^{+3}\end{array}$ & 7135 & $\begin{array}{l}1.00 \times 10^{-6} \\
4.50 \times 10^{-8}\end{array}$ & $1.05 \times 10^{-6}$ & 1.25 & $1.34 \times 10^{-6}$ & $1.45 \times 10^{-6}$ \\
\hline
\end{tabular}

\section{Results and discussion}

The adopted abundances are present in Table 5. In most instances the values derived from the combination of the
Table 4. b) Analysis of PK $359-2.3$

\begin{tabular}{lllllll}
\hline Ion & $\lambda(A)$ & $\frac{N\left(X^{+i}\right)}{N\left(H^{+}\right)}$ & $\Sigma \frac{N\left(X^{+i}\right)}{N\left(H^{+}\right)}$ & ICF & $\frac{N(X) / N(H)}{\text { ICF method }}$ & Model \\
\hline $\mathrm{H}^{+}$ & 5876,4471 & 0.1075 & 0.1161 & 1.00 & 0.116 & 0.114 \\
$H e^{++}$ & 4686,5412 & 0.0086 & & & & \\
$N^{+}$ & 6583,6548 & $6.48 \times 10^{-6}$ & $6.48 \times 10^{-6}$ & 18.4 & $1.19 \times 10^{-4}$ & $1.11 \times 10^{-4}$ \\
$O^{+}$ & 3727,7325 & $2.73 \times 10^{-5}$ & & & & \\
$O^{++}$ & 4959,5007 & $4.41 \times 10^{-4}$ & $4.68 \times 10^{-4}$ & 1.07 & $5.03 \times 10^{-4}$ & $5.20 \times 10^{-4}$ \\
$N e^{++}$ & 3868 & $4.28 \times 10^{-5}$ & $4.28 \times 10^{-5}$ & 1.14 & $4.89 \times 10^{-5}$ & $5.14 \times 10^{-5}$ \\
$S^{+}$ & 6717,6731 & $1.98 \times 10^{-7}$ & & & & \\
$S^{++}$ & 6312 & $2.43 \times 10^{-6}$ & $2.63 \times 10^{-6}$ & 2.25 & $5.91 \times 10^{-6}$ & $5.75 \times 10^{-6}$ \\
$A r^{++}$ & 7135 & $9.86 \times 10^{-7}$ & & & & \\
$A r^{+3}$ & 4740 & $4.82 \times 10^{-7}$ & $1.47 \times 10^{-6}$ & 1.81 & $2.66 \times 10^{-6}$ & $2.90 \times 10^{-6}$ \\
\hline
\end{tabular}

observed ionic abundances and the model-ICFs are preferred to those of the theoretical model. Weighted averages are adopted only in a few cases. Following the first two columns, which give the PK-designations and the usual name of the nebula, are the successive columns listing the abundances of $\mathrm{He}, \mathrm{O}, \mathrm{N}, \mathrm{Ne}, \mathrm{S}, \mathrm{Ar}$ and $\mathrm{Cl}$, presented in logarithmic form, on the scale $\log N(\mathrm{H})=12.0$. For each value we assign a letter symbol to denote the quality of the determination, as well as a measure of the expected error. The qualification of the determination is done by using the quantity $\Delta$ defined as follows:

$\Delta \equiv \frac{\left|[N(\mathrm{X}) / N(\mathrm{H})]_{\mathrm{ICF}}-[N(\mathrm{X}) / N(\mathrm{H})]_{\mathrm{MODEL}}\right|}{[N(\mathrm{X}) / N(\mathrm{H})]_{\mathrm{ICF}}}$.

The quality denoted with the alphabetical symbols should be correlated with $\Delta$ in the following ways:

$$
\begin{aligned}
\mathrm{A}: & \Delta \leq 0.20 \\
\mathrm{~B}^{+}: & 0.20<\Delta \leq 0.40 \\
\mathrm{~B}: & 0.40<\Delta \leq 0.60 \\
\mathrm{C}: & 0.60<\Delta \leq 1.0 .
\end{aligned}
$$

Results assigned with $D$ correspond to a discrepancy by a factor of slightly higher than 2 to 4 , which could mean that we have only slightly better than an order of magnitude.

The next two columns and the first, bracketed number in the last column, are related to the model of the central star surface flux distribution, which produces the best photoionization model of the nebula. The effective temperature $T_{\text {eff }}^{*}$ is given in units of $10^{3} \mathrm{~K}$, while the surface gravity $g$, in units of $\mathrm{cm} \mathrm{s}^{-2}$, is presented in logarithmic form. For almost all central stars having $T_{\text {eff }}^{*}$ above about $35000 \mathrm{~K}$, the adopted models are based on the nonLTE computation by Clegg \& Middlemass $(1987,(2))$ and Husfeld et al. $(1984,(3))$. They are extensive enough to 
Table 5. Adopted abundances for the nebulae

\begin{tabular}{|c|c|c|c|c|c|c|c|c|c|c|c|}
\hline PK No. & Name & [Be] & {$[0]$} & {$[\mathrm{N}]$} & {$[\mathrm{Ne}]$} & [S] & {$[\mathrm{Ar}]$} & {$[\mathrm{Cl}]$} & $\begin{array}{l}T_{t / f f}^{*} \\
{\left[10^{3} K\right]}\end{array}$ & $\log \mathrm{g}$ & Notes \\
\hline $0+17.1$ & Pc12 & $11.03 \mathrm{~A}$ & $8.69 \Lambda$ & $7.80 \mathrm{~A}$ & $7.68 \AA$ & $6.91 \mathrm{~A}$ & $6.13 \Lambda$ & $5.61 \mathrm{~A}$ & 37.5 & 4.50 & (2) \\
\hline $0+3.1$ & He2-250 & $11.19 \mathrm{~B}+$ & $8.32 \mathrm{~B}+$ & $8.81 \mathrm{~B}$ & $7.75 \mathrm{~B}+$ & $6.79 \mathrm{~B}+$ & $6.68 \mathrm{~B}$ & - & 85.0 & 4.75 & (3),5,8 \\
\hline $0-1.1$ & M3-43 & $11.14 \mathrm{~A}$ & $9.10 \mathrm{~B}+$ & $9.31 \mathrm{~B}$ & $8.24 \mathrm{~B}+$ & $7.23 \mathrm{~B}+$ & $7.17 \mathrm{~B}+$ & - & 102.5 & 5.25 & (2) \\
\hline $0-2.3$ & H2-32 & $11.05 \mathrm{~B}$ & $7.01 \mathrm{~B}$ & $6.30 \mathrm{C}$ & - & $5.59 \mathrm{~B}$ & - & - & 27.0 & 3.40 & (1), 5,8 \\
\hline 0.7 .1 & M2-35 & $11.10 \mathrm{~A}$ & $9.06 \AA$ & $8.63 \mathrm{~A}$ & $8.04 \mathrm{~B}+$ & $6.72 \mathrm{BH}$ & $6.84 \mathrm{~B}+$ & - & 85.0 & 5.25 & (3) \\
\hline $1-3.1$ & H $1-47$ & $11.01 \mathrm{~B}+$ & $8.01 \mathrm{~B}+$ & $7.88 \mathrm{~B}+$ & - & $6.62 \mathrm{~B}+$ & $\longrightarrow$ & - & 30.0 & 4.50 & $(1), 7$ \\
\hline 1.4 .2 & H1-56 & $11.04 \mathrm{~A}$ & $8.73 \AA$ & $8.24 \mathrm{~B}+$ & $7.84 \mathrm{~A}$ & $6.90 \mathrm{~B}$ & $6.39 \mathrm{~B}+$ & 一 & 65.0 & 4.75 & (2) \\
\hline $2+8.1$ & H1-11 & $10.98 \mathrm{~A}$ & $8.96 \mathrm{~A}$ & $8.01 \mathrm{~B}+$ & $8.29 \mathrm{~B}+$ & $7.14 \mathrm{~B}+$ & $6.56 \mathrm{~B}+$ & 一 & 80.0 & 4.70 & $(2), 6$ \\
\hline $2+1.1$ & H2-20 & $11.04 \mathrm{~B}+$ & $9.20 \mathrm{~B}$ & $8.61 \mathrm{~B}+$ & - & $6.72 \mathrm{~B}$ & $6.48 \mathrm{~B}$ & - & 27.5 & 3.30 & (1),5,8 \\
\hline $2-1.1$ & Pe2-1 & $11.21 \mathrm{~A}$ & $8.47 \mathrm{~B}+$ & $8.84 \mathrm{~B}+$ & $7.72 \mathrm{~B}$ & $6.68 \mathrm{~B}$ & $6.59 \mathrm{~B}$ & - & 47.5 & 4.70 & $(2), 5$ \\
\hline $2-2.4$ & M2-23 & $10.98 \AA$ & $8.22 \Lambda$ & $7.40 \mathrm{~B}+$ & $7.15 \mathrm{~A}$ & $6.30 \mathrm{BH}$ & $5.81 \mathrm{~A}$ & $5.62 \mathrm{~B}+$ & 57.5 & 4.00 & (2),9 \\
\hline $2-3.2$ & H2-37 & $11.10 \mathrm{~B}+$ & $8.73 \mathrm{~A}$ & $8.30 \mathrm{~B}+$ & $8.10 \mathrm{~B}+$ & $6.85 \mathrm{~B}+$ & $6.47 \mathrm{~A}$ & - & 52.5 & 5.60 & (2) \\
\hline 2.3 .3 & $M 1-37$ & $11.11 \mathrm{~B}+$ & $7.98 \mathrm{~B}+$ & $8.10 \mathrm{~A}$ & - & $6.58 \mathrm{~B}$ & $6.52 \mathrm{~B}$ & - & 29.0 & 3.90 & (i) \\
\hline $2-3.5$ & M1-38 & $11.04 \mathrm{~B}+$ & $9.00 \mathrm{~B}+$ & $8.21 \mathrm{~B}+$ & $7.98 \mathrm{~B}$ & $6.97 \mathrm{~B}$ & $6.24 \mathrm{~B}+$ & 一 & 27.0 & 3.20 & (b) $, 5,9$ \\
\hline $2 \cdot 3.6$ & H2-39 & $11.09 \mathrm{~A}$ & $8.35 \mathrm{~A}$ & $8.00 \mathrm{~B}+$ & - & - & $6.18 \mathrm{~B}+$ & 一 & 100.0 & 5.00 & (2),6 \\
\hline $2-4.1$ & H1-54 & $10.99 \mathrm{~B}+$ & $8.56 \mathrm{~B}+$ & $7.48 \mathrm{~B}+$ & $7.49 \mathrm{~B}$ & $6.81 \mathrm{~B}+$ & $6.12 \mathrm{~B}+$ & $=$ & 42.5 & 4.25 & $(2), 9$ \\
\hline $2-4.2$ & M1-42 & $11.22 \mathrm{~A}$ & $8.57 \mathrm{~A}$ & $8.75 \mathrm{~A}$ & $8.03 \mathrm{~A}$ & $7.16 \AA$ & $6.58 \mathrm{~A}$ & $5.80 \mathrm{~A}$ & 100.0 & 5.25 & $(2), 9$ \\
\hline $2-6.1$ & M2-33 & $10.96 \mathrm{~A}$ & $8.67 \mathrm{~A}$ & $7.65 \mathrm{~B}+$ & $7.83 \mathrm{~B}+$ & $6.72 \mathrm{~A}$ & $6.33 \Lambda$ & - & 55.0 & 4.20 & (2) \\
\hline $2-9.1$ & Cn1-5 & $11.06 \mathrm{~A}$ & $9.05 \mathrm{~A}$ & $8.63 \mathrm{~B}+$ & $8.10 \mathrm{~B}$ & $7.50 \mathrm{~A}$ & $7.03 \mathrm{~B}+$ & $5.91 \mathrm{~B}$ & 62.5 & 5.20 & (2) \\
\hline $3+5.1$ & $\mathrm{H} 2.15$ & $11.15 \mathrm{~A}$ & $8.86 \mathrm{~A}$ & $9.19 \mathrm{~B}+$ & $8.24 \mathrm{~B}+$ & $7.49 \mathrm{~B}+$ & $6.98 \wedge$ & - & 95.0 & 5.25 & $(2), 8$ \\
\hline $3+3.1$ & H2-17 & $10.95 \mathrm{~B}$ & 7.55 B & $7.77 \mathrm{~B}+$ & $7.33 \mathrm{~B}$ & $0.12 \mathrm{~B}$ & $\cdots$ & 一 & 35.5 & 4.50 & $(2), 5,8$ \\
\hline $3-4.3$ & H1-59 & $11.01 \mathrm{~A}$ & $8.58 \mathrm{~B}+$ & $8.67 \mathrm{~B}$ & $7.79 \mathrm{~B}+$ & $7.52 \mathrm{~B}$ & $6.63 \mathrm{~B}+$ & - & 125.0 & 5.50 & (2) \\
\hline $3-4.4$ & H2-41 & $11.33 \mathrm{~B}+$ & $8.07 \mathrm{~B}$ & $8.41 \mathrm{~B}$ & $7.42 \mathrm{~B}+$ & $6.54 \mathrm{~B}+$ & $6.32 \mathrm{~B}$ & - & 80.0 & 4.50 & (2),7,9 \\
\hline $3-4.7$ & Ap $1-12$ & $11.07 \mathrm{~B}+$ & $8.31 \mathrm{~B}+$ & $8.03 \mathrm{~B}+$ & $7.52 \mathrm{~B}$ & $6.59 \mathrm{~B}+$ & - & - & 29.0 & 3.40 & $(1), 7$ \\
\hline 3-4.9 & $112-43$ & $11.01 \mathrm{~B}$ & $6.90 \mathrm{D}$ & $6.80 \mathrm{D}$ & - & $5.75 \mathrm{C}$ & $5.01 \mathrm{C}$ & - & 77.5 & 4.50 & (2) \\
\hline $3-6.1$ & M2-36 & $11.09 \mathrm{~A}$ & $8.89 \mathrm{~A}$ & $8.62 \mathrm{~B}+$ & $8.27 \mathrm{~B}$ & $7.17 \mathrm{~B}+$ & $6.61 \mathrm{~B}+$ & $5.98 \mathrm{~B}+$ & 82.5 & 5.20 & (2) \\
\hline $3-17.1$ & Hb8 & $10.95 \mathrm{~A}$ & $8.68 \mathrm{~A}$ & $7.91 \mathrm{~A}$ & $7.95 \Lambda$ & $6.75 \mathrm{~A}$ & $6.20 \Lambda$ & $5.29 \mathrm{~B}+$ & 75.0 & 4.75 & (3) \\
\hline $4+6.2$ & H1-24 & $11.17 \mathrm{~A}$ & $7.52 \mathrm{~B}+$ & $7.71 \Lambda$ & $6.62 \mathrm{~B}$ & $6.45 \mathrm{~B}+$ & $6.00 \mathrm{~B}+$ & - & 40.0 & 4.70 & $(2), 7$ \\
\hline $4+4.1$ & M1-25 & $11.10 \mathrm{~A}$ & $9.09 \mathrm{~A}$ & $8.82 \mathrm{~A}$ & $8.41 \mathrm{D}+$ & $7.47 \mathrm{~A}$ & $6.92 \mathrm{~B}+$ & $5.99 \mathrm{~B}+$ & 47.5 & 4.20 & (2) \\
\hline $4-3.1$ & M2-29 & $11.11 \mathrm{~A}$ & $7.47 \mathrm{~A}$ & $7.01 \mathrm{~B}+$ & $6.81 \mathrm{~B}$ & $5.83 \mathrm{~B}+$ & $5.64 \mathrm{Bt}$ & - & 50.0 & 4.00 & (2) \\
\hline $355-2.3$ & H1-32 & $10.95 \mathrm{~B}+$ & $8.12 \mathrm{~B}+$ & $7.59 \mathrm{~B}+$ & $7.27 \mathrm{~B}$ & $6.19 \mathrm{~B}$ & $5.95 \mathrm{~B}+$ & - & 35.5 & 3.70 & $(2), 9$ \\
\hline $355-2.4$ & H1-31 & $11.07 \mathrm{~A}$ & $8.86 \mathrm{~A}$ & $8.30 \mathrm{~A}$ & $8.04 \mathrm{~A}$ & $6.96 \mathrm{~B}+$ & $6.53 \AA$ & 一 & 77.5 & 4.70 & $(2), 9$ \\
\hline $355 \cdot 3.3$ & H1-35 & $10.98 \Lambda$ & $8.31 \mathrm{~A}$ & $7.39 \Lambda$ & $7.60 \mathrm{~B}$ & $6.58 \mathrm{~A}$ & $6.17 \mathrm{~B}$ & - & 47.5 & 4.70 & (2) \\
\hline $355-4.2$ & MI-30 & $11.12 \mathrm{~B}+$ & $8.55 \mathrm{~B}+$ & $9.00 \Lambda$ & $7.37 \mathrm{~B}+$ & $7.29 \mathrm{~B}+$ & $6.94 \mathrm{~B}+$ & $5.81 \AA$ & 65.0 & 4.75 & (2) \\
\hline $355-6.1$ & M3-21 & $11.05 \AA$ & $8.74 \Lambda$ & $8.26 \wedge$ & $8.00 \AA$ & $7.02 \Lambda$ & $0.58 \mathrm{~A}$ & $5.77 \mathrm{~B}+$ & 82.5 & 4.70 & (2) \\
\hline $356+4.1$ & M2-11 & $11.08 \AA$ & $8.81 \AA$ & $8.03 \mathrm{~B}+$ & $7.36 \mathrm{~B}+$ & $6.99 \mathrm{~B}+$ & $6.36 \mathrm{~B}+$ & - & 140.0 & 一 & (4) \\
\hline $356+4.2$ & M3-38 & $11.11 \mathrm{~A}$ & $8.37 \AA$ & $8.69 \mathrm{~B}+$ & $7.73 \mathrm{~B}+$ & $6.90 \mathrm{~B}+$ & $6.36 \mathrm{~B}+$ & - & 155.0 & - & (1) \\
\hline $356-3.1$ & H2-26 & $11.20 \mathrm{~A}$ & $8.49 \mathrm{~B}+$ & $8.87 \mathrm{~B}+$ & $7.80 \mathrm{~B}$ & $6.96 \mathrm{~B}+$ & $6.85 \mathrm{~B}$ & - & 87.5 & 5.20 & (2),8 \\
\hline $356-4.1$ & Cn2-1 & $10.98 \AA$ & $9.23 \mathrm{~A}$ & $8.70 \mathrm{~B}+$ & $8.55 \mathrm{~A}$ & $7.47 \mathrm{~A}$ & $6.76 \mathrm{~B}+$ & $5.65 \mathrm{~B}+$ & 50.0 & 3.80 & (2) \\
\hline $356-5.2$ & M2-24 & $11.13 \mathrm{~B}+$ & $7.90 \mathrm{~B}$ & $7.94 \mathrm{~B}+$ & $7.56 \mathrm{~B}$ & $6.45 \mathrm{~B}$ & $6.00 \mathrm{~B}$ & - & 55.0 & 5.20 & (2) \\
\hline $357+7.1$ & $M 4-3$ & $10.98 \Lambda$ & $8.67 \AA$ & $8.08 \mathrm{~B}+$ & $7.94 \mathrm{~B}+$ & $7.03 \mathrm{~B}+$ & $6.23 \mathrm{~A}$ & 一 & 57.5 & 4.25 & (2) \\
\hline $357+4.1$ & 112-7 & $11.09 \mathrm{~A}$ & $8.56 \AA$ & $7.82 \AA$ & $7.79 \mathrm{~B}+$ & $6.01 \mathrm{~B}+$ & $6.31 \mathrm{~B}+$ & - & $\mathbf{5 0 . 0}$ & 4.50 & (2) \\
\hline $357+3.1$ & M3-7 & $11.00 \mathrm{~A}$ & $8.69 \mathrm{~A}$ & $7.81 \mathrm{~B}+$ & $7.63 \mathrm{~A}$ & $6.91 \mathrm{~B}+$ & $6.57 \mathrm{~A}$ & - & 80.0 & 4.70 & (2) 6,9 \\
\hline $357+3.2$ & M3-41 & $11.10 \mathrm{~B}+$ & $8.09 \mathrm{~B}+$ & $8.16 \mathrm{~B}+$ & $7.90 \mathrm{~B}$ & $6.75 \mathrm{~B}+$ & $5.99 \mathrm{~B}$ & - & 28.0 & 3.20 & (1) \\
\hline $357+3.4$ & M3-42 & $11.08 \mathrm{~A}$ & $9.09 \mathrm{~B}+$ & $8.98 \mathrm{~B}$ & 8.71 B & $7.27 \mathrm{~B}$ & $7.01 \mathrm{~B}$ & - & 120.0 & 5.70 & $(2), 6,9$ \\
\hline $357+2.4$ & H1-18 & $11.22 \Lambda$ & $8.94 \mathrm{D}+$ & $9.03 \mathrm{~B}+$ & $8.22 \mathrm{~B}+$ & $7.22 \mathrm{~B}+$ & $6.95 \Lambda$ & 一 & 65.0 & 4.75 & (2) \\
\hline $357+1.1$ & H1-23 & $11.13 \mathrm{~A}$ & $8.78 \mathrm{~A}$ & $8.54 \mathrm{~B}+$ & $8.11 \mathrm{~B}+$ & $7.07 \mathrm{~B}+$ & $6.76 \mathrm{~A}$ & - & 52.5 & 4.00 & (2),8,9 \\
\hline $357-3.2$ & M2-16 & $11.11 \mathrm{~A}$ & $8.77 \AA$ & $8.47 \mathrm{~B}+$ & $8.44 \mathrm{~B}+$ & $7.08 \mathrm{~B}+$ & $6.68 \mathrm{~A}$ & $5.49 \mathrm{~B}+$ & 102.5 & 5.40 & $(2), 6$ \\
\hline $357-4.2$ & M2-22 & $11.17 \AA$ & $8.59 \mathrm{~A}$ & $8.95 \mathrm{~B}$ & $8.30 \mathrm{~B}$ & $7.03 \mathrm{~B}+$ & $6.66 \mathrm{~B}+$ & - & 82.0 & 4.75 & $(2), 9$ \\
\hline 357.5 .1 & M1-34 & $11.12 \mathrm{~A}$ & $9.03 \mathrm{~A}$ & $8.65 \mathrm{~B}+$ & - & $7.61 \mathrm{~B}+$ & $6.87 \mathrm{~B}+$ & $\cdots$ & 105.0 & 5.70 & (2) \\
\hline $357-6.1$ & M3.50 & $11.11 \mathrm{~A}$ & $9.07 \mathrm{Bt}$ & $9.32 \mathrm{~B}$ & $8.16 \mathrm{~B}+$ & $7.61 \mathrm{~B}$ & $7.06 \mathrm{~B}+$ & - & 122.5 & 5.70 & (2) \\
\hline $358+7.1$ & M3-36 & $10.99 \mathrm{~A}$ & $8.75 \mathrm{~A}$ & - & $8.05 \mathrm{~B}$ & $6.90 \mathrm{~B}+$ & $6.45 \mathrm{~B}+$ & $5.92 \mathrm{~B}+$ & 65.0 & 4.75 & $(2), 6$ \\
\hline $358+4.1$ & M3-8 & $11.11 \mathrm{~B}+$ & $8.02 \mathrm{~B}+$ & $8.75 \mathrm{~A}$ & $8.20 \mathrm{~B}$ & $6.54 \mathrm{~B}+$ & $6.68 \mathrm{~B}+$ & - & 70.0 & 5.00 & $(2), 9$ \\
\hline $358+3.1$ & M3-10 & $11.04 \AA$ & $8.89 \mathrm{~A}$ & $8.22 \mathrm{~B}+$ & $7.96 \mathrm{~B}+$ & $6.82 \mathrm{~B}+$ & $6.40 \mathrm{~B}+$ & 一 & 102.5 & 5.70 & (2) \\
\hline $358+3.6$ & H1-20 & $11.07 \mathrm{~A}$ & $8.97 \AA$ & $8.74 \mathrm{~A}$ & $8.71 \mathrm{~B}+$ & $6.56 \mathrm{~B}$ & $6.75 \mathrm{~B}+$ & $5.97 \mathrm{~B}$ & 65.0 & 4.75 & (2),6,8 \\
\hline $358+3.7$ & H1-17 & $11.00 \mathrm{~A}$ & $8.61 \mathrm{~A}$ & 8.32 B+ & - & $6.76 \mathrm{~B}+$ & $6.42 \mathrm{~A}$ & - & 77.5 & 4.75 & $(2), 0,8$ \\
\hline $358+1.1$ & M4-6 & $10.98 \mathrm{~A}$ & $8.31 \mathrm{~B}+$ & $7.97 \mathrm{~B}+$ & $7.62 \mathrm{~B}$ & $6.34 \mathrm{C}$ & $6.22 \mathrm{~B}$ & - & 125.0 & 6.75 & $(2), 5,8$ \\
\hline 358.3 .1 & II1-44 & $11.10 \mathrm{~B}+$ & $8.68 \mathrm{~B}+$ & $8.52 \mathrm{~B}+$ & $二$ & $6.59 \mathrm{~B}$ & $6.57 \mathrm{~B}$ & - & 30.0 & 3.70 & (1),8 \\
\hline $359+4.1$ & Th3-14 & $11.05 \mathrm{~B}+$ & $8.01 \mathrm{~B}+$ & $7.80 \mathrm{~A}$ & $\longrightarrow$ & $6.25 \mathrm{~B}+$ & $5.37 \mathrm{~B}+$ & - & 32.5 & 4.20 & $(1), 8$ \\
\hline $359+3.4$ & $A 12-E$ & $11.02 \mathrm{~A}$ & $8.69 \mathrm{~B}+$ & $8.44 \mathrm{~B}$ & $7.94 \mathrm{~B}+$ & $6.36 \mathrm{~B}$ & $6.34 \mathrm{~B}+$ & 一 & 130.0 & 5.70 & (2) \\
\hline $359-1.1$ & $M 1-29$ & $11.14 \mathrm{~A}$ & $9.00 \AA$ & $8.82 \mathrm{~B}+$ & $8.31 \mathrm{~B}+$ & $7.42 \mathrm{B+}$ & $6.92 \mathrm{~A}$ & $5.72 \mathrm{~B}+$ & 102.5 & 5.25 & (2) \\
\hline $359-1.3$ & M3-45 & $11.02 \mathrm{~A}$ & $8.48 \mathrm{~B}+$ & $7.87 \mathrm{~B}$ & $7.58 \mathrm{~B}$ & $6.59 \mathrm{~B}$ & $6.13 \mathrm{~B}$ & $\longrightarrow$ & 120.0 & 二 & (4),8 \\
\hline $359-2.3$ & H1-40 & $11.06 \AA$ & $8.70 \mathrm{~A}$ & $8.08 \mathrm{~A}$ & $7.69 \AA$ & $6.77 \AA$ & $6.43 \mathrm{~A}$ & - & 80.0 & 4.70 & (2) \\
\hline $359-2.4$ & M3-46 & $11.09 \mathrm{~B}+$ & $8.42 \mathrm{~B}+$ & $8.69 \mathrm{~A}$ & $7.68 \mathrm{~B}+$ & $7.08 \mathrm{~B}+$ & $6.47 \mathrm{~B}$ & - & 75.0 & 4.70 & (3),5,8,9 \\
\hline $359-4.2$ & $M 2.27$ & $11.07 \mathrm{~B}+$ & $8.77 \mathrm{~B}+$ & $8.46 \mathrm{~A}$ & $8.19 \mathrm{~B}$ & $7.17 \mathrm{Bt}$ & $6.77 \mathrm{~B}+$ & 一 & 40.0 & 4.80 & (2) \\
\hline
\end{tabular}

allow us to make interpolations or slight extrapolations in $T_{\text {eff }}^{*}$ and $\log g$. For some very high $T_{\text {eff }}^{*}$ nebulae $(\mathrm{M} 2-11$, M3 - 38 and M3 - 45) the Planckian distribution (4) can reproduce the spectra better than the non-LTE model atmospheres. The Kurucz LTE-models $(1979,(1))$ are used in the nebulae with $T_{\text {eff }}^{*}$ lower than $35000 \mathrm{~K}$.
Next, we will discuss the individual elements. The discussion on the errors introduced by the observation has been given in Sects. 2 and 3.2. In the following, we will mainly stress the role of various aspects related to the derivation of ICFs in evaluating the expected average uncertainties in the finally adopted abundances. For the discussion, the ionization structures of PK $0+17.1$ with 
Table 5. continued

\begin{tabular}{|c|c|c|c|c|c|c|c|c|c|c|c|}
\hline PK No. & Name & [He] & {$[0]$} & [N] & {$[\mathrm{Ne}]$} & {$[\mathrm{S}]$} & [Ar] & {$[\mathrm{Cl}]$} & $\begin{array}{l}T_{e f f}^{\star} \\
\left\{10^{3} K\right\}\end{array}$ & $\log g$ & Noles \\
\hline $4-11.1$ & M3-29 & $10.95 \mathrm{~A}$ & $8.50 \mathrm{D}+$ & $7.98 \mathrm{~A}$ & $7.18 \mathrm{~B}+$ & $6.14 \mathrm{~A}$ & $6.16 \mathrm{~A}$ & - & 40.0 & 4.50 & (2) \\
\hline $5+4.1$ & H $1-27$ & $11.13 \mathrm{~B}+$ & $8.42 \mathrm{~A}$ & $8.47 \mathrm{~B}+$ & $7.60 \mathrm{~B}+$ & - & $6.66 \mathrm{~B}+$ & - & 50.0 & 5.20 & (2) \\
\hline $5 \cdot 3.1$ & H1-58 & $10.98 \mathrm{~B}+$ & $8.11 \mathrm{~A}$ & $7.11 \mathrm{~A}$ & - & $6.34 \mathrm{~B}+$ & $5.73 \mathrm{~B}+$ & - & 50.0 & 4.50 & (2) \\
\hline $6+8.1$ & MI-20 & $11.02 \mathrm{~A}$ & $8.62 \mathrm{~A}$ & $7.75 \AA$ & $7.79 \mathrm{~B}+$ & $6.52 \mathrm{~B}$ & $6.05 \mathrm{~B}+$ & $5.06 \mathrm{~B}$ & 50.0 & 4.60 & (2),9 \\
\hline $6+4.2$ & M3-15 & $11.03 \mathrm{~A}$ & $8.74 \mathrm{~A}$ & $8.14 \mathrm{~A}$ & $7.86 \mathrm{~A}$ & 6. $86 \mathrm{~B}+$ & $6.53 \mathrm{~B}+$ & - & 72.5 & 4.70 & (2) \\
\hline $6+4.1$ & 112-18 & $10.99 \mathrm{~A}$ & $8.76 \mathrm{~A}$ & 8.36 B+ & $7.87 \mathrm{~B}+$ & $6.84 \mathrm{~B}+$ & $6.33 \mathrm{~B}+$ & - & 82.5 & 5.00 & (2), 5 \\
\hline $6-3.3$ & M2-31 & $10.95 \mathrm{~A}$ & $8.68 \mathrm{~A}$ & $8.17 \mathrm{~A}$ & $8.05 \mathrm{~B}+$ & $6.93 \mathrm{~B}$ & $6.37 \mathrm{~B}+$ & - & 60.0 & 4.70 & (2) \\
\hline $7+7.1$ & M1-22 & $11.25 \AA$ & $8.34 \mathrm{~A}$ & $8.24 \mathrm{~B}+$ & $7.43 \mathrm{~B}+$ & $6.86 \mathrm{~B}+$ & $6.40 \mathrm{~A}$ & - & 77.5 & 4.60 & (2),5 \\
\hline $7-4.1$ & H11-65 & $11.10 \mathrm{~B}+$ & $8.55 \mathrm{~B}+$ & $8.45 \mathrm{~B}+$ & $\longrightarrow$ & $6.87 \mathrm{Bt}$ & - & - & 27.5 & 3.80 & $(1), 5$ \\
\hline $7-6.1$ & 111.66 & $11.17 \AA$ & $8.47 \AA$ & $8.13 \mathrm{Bt}$ & $7.23 \mathrm{~B}+$ & $6.69 \mathrm{~B}+$ & $6.45 \mathrm{~B}+$ & - & 100.0 & 5.20 & (3) \\
\hline $7-6.2$ & Vy2-1 & $11.03 \mathrm{~A}$ & $8.76 \mathrm{~A}$ & $7.98 \Lambda$ & $8.07 \mathrm{~A}$ & $7.01 \AA$ & $6.54 \mathrm{~B}+$ & $5.44 \mathrm{~B}$ & 60.0 & 5.00 & (2) \\
\hline 8-4.2 & M2-42 & $11.05 \mathrm{~A}$ & $8.20 \mathrm{~A}$ & $7.75 \mathrm{~B}+$ & $6.90 \mathrm{~B}$ & $6.62 \mathrm{~B}+$ & $6.06 \mathrm{~B}+$ & $5.62 \mathrm{~B}+$ & 50.0 & 4.70 & (2) \\
\hline 8.7 .1 & He2-406 & $11.18 \mathrm{~A}$ & $9.08 \mathrm{~A}$ & $8.63 \mathrm{~B}+$ & $\longrightarrow$ & $7.48 \mathrm{Bt}$ & $7.03 \mathrm{~B}+$ & - & 102.5 & 5.50 & (2), 5 \\
\hline $8-7.2$ & NGC6644 & $11.03 \mathrm{~A}$ & $8.54 \mathrm{~A}$ & $8.00 \mathrm{~A}$ & $7.85 \mathrm{~B}+$ & $6.40 \mathrm{Bt}$ & $6.12 \mathrm{~A}$ & $4.99 \mathrm{~B}+$ & 125.0 & 6.75 & (2) \\
\hline $9-10.1$ & M3.33 & $10.98 \mathrm{~A}$ & $8.45 \mathrm{~A}$ & $7.38 \mathrm{~B}+$ & $7.75 \mathrm{~B}+$ & - & $6.08 \mathrm{~A}$ & - & 82.5 & 4.75 & $(2), 6$ \\
\hline $11+11.1$ & M2-13 & $11.03 \mathrm{~A}$ & $8.92 \mathrm{~A}$ & $8.13 \mathrm{~A}$ & $7.53 \mathrm{~B}+$ & $7.15 \mathrm{~B}+$ & $6.58 \mathrm{~B}+$ & - & 52.5 & 5.70 & (2) \\
\hline $12-12.1$ & M1-45 & $11.05 \mathrm{D}+$ & $8.73 \mathrm{~B}+$ & 8.30 B+ & - & $6.96 \mathrm{Bt}$ & $\longrightarrow$ & 一 & 29.0 & 3.60 & $(1), 5,8$ \\
\hline $13-4.1$ & Pe2-14 & $11.28 \mathrm{~A}$ & $8.28 \mathrm{~A}$ & $7.19 \mathrm{~B}+$ & - & $6.68 \mathrm{Bt}$ & $6.35 \mathrm{~B}+$ & 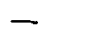 & 110.0 & 5.80 & (2) \\
\hline $14-4.1$ & M1-50 & $11.06 \mathrm{~A}$ & $8.73 \Lambda$ & $7.75 \mathrm{~A}$ & $7.86 \mathrm{~B}+$ & $6.77 \mathrm{\Lambda}$ & $6.45 \AA$ & 一 & 100.0 & 5.25 & (3) \\
\hline $15-4.1$ & M1-53 & $11.06 \mathrm{~A}$ & $8.61 \mathrm{~A}$ & - & $7.26 \mathrm{~B}+$ & - & $6.16 \mathrm{~B}+$ & - & 77.5 & 4.70 & $(2), 6$ \\
\hline $16-4.1$ & M1.54 & $11.08 \AA$ & $8.98 \mathrm{~A}$ & $8.82 \AA$ & $7.90 \AA$ & $7.40 \mathrm{~A}$ & $6.81 \Lambda$ & $5.62 \mathrm{Bt}$ & 85.0 & 4.80 & (2) \\
\hline $16-4.2$ & M1-56 & $11.03 \mathrm{~A}$ & $8.76 \mathrm{~A}$ & 8.10 B+ & $7.91 \mathrm{~B}+$ & $6.95 \mathrm{D}+$ & $6.44 \mathrm{~A}$ & $5.30 \mathrm{B+}$ & 80.0 & 4.70 & (2) \\
\hline $345+6.1$ & $\mathrm{He} 2-175$ & $11.16 \mathrm{~A}$ & $9.00 \mathrm{~A}$ & $8.59 \mathrm{~B}+$ & - & $7.50 \mathrm{~B}+$ & $6.92 \mathrm{~B}+$ & - & 105.0 & 5.50 & (2) \\
\hline $346+8.1$ & He2-171 & $11.04 \mathrm{~A}$ & $7.79 \mathrm{~B}+$ & $7.62 \mathrm{~B}+$ & $7.49 \mathrm{~B}+$ & $6.50 \mathrm{~B}$ & $5.76 \mathrm{Bt}$ & $=$ & 110.0 & 5.15 & (2) \\
\hline $349+4.1$ & M2-4 & $10.99 \mathrm{~B}+$ & $8.80 \mathrm{~B}+$ & $8.09 \mathrm{~A}$ & $7.90 \mathrm{~B}$ & $6.96 \mathrm{~B}$ & $6.25 \mathrm{~B}$ & 一 & 50.0 & 4.75 & (2) \\
\hline $351+5.1$ & M2-5 & $11.07 \mathrm{~B}+$ & $8.35 \mathrm{~B}+$ & $8.68 \mathrm{~A}$ & - & $7.30 \mathrm{~B}$ & $6.46 \mathrm{~B}+$ & - & 37.5 & 4.25 & (2) \\
\hline $353+6.2$ & $M 2-6$ & $10.95 \mathrm{~A}$ & $8.73 \mathrm{~A}$ & $7.74 \mathrm{~B}+$ & $7.93 \mathrm{DH}$ & $6.77 \mathrm{~B}+$ & $6.12 \mathrm{~A}$ & - & 57.5 & 5.20 & $(2), 8$ \\
\hline $354+4.1$ & M2-10 & $11.01 \mathrm{~B}+$ & $8.86 \mathrm{~B}+$ & $8.37 \mathrm{~A}$ & $8.20 \mathrm{~B}$ & $6.85 \mathrm{~B}+$ & $6.58 \mathrm{~B}$ & 5.32. B & 35.5 & 4.20 & $(2), 5$ \\
\hline $355-2.1$ & M3-14 & $11.18 \mathrm{~A}$ & $9.03 \mathrm{~A}$ & $8.78 \mathrm{~B}+$ & - & $7.09 \mathrm{~B}+$ & $6.89 \mathrm{~B}+$ & - & 97.5 & 5.25 & (2) \\
\hline $355-2.2$ & H1-29 & $11.04 \mathrm{~A}$ & $8.45 \mathrm{~A}$ & $7.87 \mathrm{~B}+$ & - & $6.29 \mathrm{~B}+$ & $6.28 \mathrm{~B}+$ & - & 57.5 & 4.72 & (2) \\
\hline
\end{tabular}

Notes : (1) Kurucz (1979), (2) Clegg and Middlemass (1987), (3) Husfeld et al. (1981), (4)

Planckian distribution. (5) The derived electron temperature, $T_{c}$, is uncertain. (6) The electron density

$N_{e}$, is derived from [ArIV] doublet. (7) The adopted electron density is uncertain. (8) The faintness, due

to the very large interstellar extinction and/or due to the intringically low surface brightness of the nebula,

causes the line intensities blueward of $H \rho$ to be poorly determined. (9) The observed line intensity ratio of

[OII] $\lambda 3727 / \lambda 3725$ cannot be reproduced by the model. The line intensity at $\lambda 7325$ predicted by the model

excitation class 3.5 and PK $359-2.3$ with excitation class 6 shown in Figs. 4b, c and 5b, c, should be used as guides.

Helium As has been shown in the case of PK $0+17.1$, in the low excitation nebulae the helium abundance determination is handicapped by the presence of neutral helium. If the fraction of $\mathrm{He}^{+}$is much less than about $30 \%$, we relied on the model calculation and the adopted helium abundance should be considered as a tentative result. In all computation involving $\mathrm{He}$ II $\lambda$ 4686 , we have assumed that the line is of nebular origin. While this might not be true in some cases, it would be extremely difficult to subtract the nonnebular emission in the present spectra of bulge PNe. We estimate the uncertainties in the low excitation nebulae to be $20 \%$ to $30 \%$. In the medium and high excitation nebulae the values should be less than about $10 \%$.

Oxygen Compared with other elements, excluding helium, oxygen has generally the best determined abundance. In a low excitation nebula where the fraction of $\mathrm{O}^{+2}$ usually drops far below $50 \%$, the lines coming from the more dominant state $\left(\mathrm{O}^{+}\right)$can be easily detected. In the other extreme, when the degree of excitation is very high (excitation class $\geq 7.5$ ) although the lines originating from stages higher than $\mathrm{O}^{+2}$ are not available, a reasonably good abundance can still be obtained because the fraction of $\mathrm{O}^{+2}$ is usually still high enough (typically 30\%-60\%). We can assign a typical uncertainty of about $30 \%$ for the oxygen abundances for the whole range of excitation.

Nitrogen In general, for nebulae without He II $\lambda 4686$ emission, nitrogen abundances can be determined with a reasonable precision. In very high excitation nebulae, in which the fraction of $\mathrm{N}^{+}$is typically much lower than $10 \%$ the total abundance could be in error by a large factor. We expect errors of about $25 \%, 50 \%$ and $75 \%$ in the cases of, respectively, low, medium and high excitation nebulae.

Neon The fractional properties of the most frequently observed stage of neon $\left(\mathrm{Ne}^{+2}\right)$ are comparable to those of $\mathrm{O}^{+2}$. However, in contrast to oxygen, in the low and very high excitation nebulae where the fraction of $\mathrm{Ne}^{+2}$ is low, the more dominant stages $\left(\mathrm{Ne}^{+}\right.$in the low, and $\mathrm{Ne}^{\geq+3}$ in the high excitation nebulae) cannot be seen in the spectra analysed. One must extend the spectral coverage toward either far-ultraviolet or farinfrared regions in order to get access to those ions. We estimate the uncertainties in the medium and high excitation cases to be about $30 \%$ to $45 \%$, while in the 
Table 6. Abundances from the literature

\begin{tabular}{|c|c|c|c|c|c|c|c|c|c|c|c|}
\hline PK No. & Name & [He] & [0] & {$[\mathrm{N}]$} & {$[\mathrm{Ne}]$} & [S] & {$[\mathrm{Ar}]$} & {$[\mathrm{Cl}]$} & $\left.\begin{array}{l}T_{j J}^{* \prime} \\
{\left[10^{3}\right.}\end{array}\right]$ & $\log B$ & Notes* \\
\hline $0-2.4$ & M2-21 & 11.10 & 8.49 & 7.95 & 7.70 & 0.01 & 6.01 & - & 82.5 & 4.72 & (3) \\
\hline $2 \cdot 2.2$ & M3-20 & 10.99 & 8.70 & 7.73 & 8.00 & 0.74 & 6.08 & 5.21 & $\mathbf{5 0 . 0}$ & 3.80 & (3) \\
\hline 3-2.1 & ML-35 & 11.21 & 8.48 & 8.79 & 7.63 & 6.75 & 6.63 & - & 62.5 & 4.78 & (3), 5 \\
\hline $3-2.3$ & IC4673 & 11.16 & 8.72 & 8.45 & 8.15 & 7.26 & $6.90:$ & - & 107.5 & 5.15 & (3), 9 \\
\hline $3-4.8$ & M2-30 & 11.08 & 8.75 & 8.06 & 8.07 & 7.11 & 6.68 & $\longrightarrow$ & 100.0 & 4.72 & (3), 9 \\
\hline $5 \cdot 6.1$ & NGC6620 & 11.06 & 8.84 & 8.65 & 8.18 & 7.10 & 6.60 & 5.48 & 85.0 & 4.72 & (3) \\
\hline $10-6.1$ & IC4732 & 11.03 & 8.42 & 7.94 & 7.71 & 6.79 & 5.98 & 4.90 & 62.5 & 4.72 & $(3), 9$ \\
\hline $11+5.1$ & NGC6439 & 11.13 & 8.65 & 8.46 & 8.00 & 8.00 & 6.45 & 5.59 & 80.0 & 4.72 & (3) \\
\hline
\end{tabular}

- see the notes for Table 5 .

low excitation nebulae the values are between $40 \%$ and $60 \%$.

Sulfur The uncertainties in the abundance determinations come mainly from the fact that we generally do not observe the predominant ions. The bulk of sulfur in $\mathrm{PNe}$ are in the form of $\mathrm{S}^{+2}$ and $\mathrm{S}^{+3}$ (Pottasch et al. 1986). While the lines originating from $\mathrm{S}^{+3}$ are not available, the line mostly used in deriving $\mathrm{N}\left(\mathrm{S}^{+2}\right)$ at $\lambda 6312 \AA$ is subject to a large uncertainty as it is usually weak and very sensitive to the adopted $T_{\varepsilon}$. The relatively well-observed ion $\mathrm{S}^{+}$contributes, in general, only about $10 \%$ or less to the total abundance, and thus needs a very large (and very uncertain) ICF, if it is the only ion observed. The uncertainties expected for sulfur abundances should be between $30 \%$ and $60 \%$.

Argon A more extensive discussion on abundance determination of this element has been given by Ratag \& Pottasch (1990). The main problems are (a) the effective collision strengths of $\mathrm{Ar}^{+3}$, one of the very important ions in the medium and high excitation nebulae, are strongly dependent on electron temperature, (b) as the $\mathrm{Ar}^{+3}$ ion is involving a $3 \mathrm{p}^{3}$-configuration, the ionic concentration can be strongly affected by the presence of density fluctuation, which is extremely difficult to handle in theoretical models, and (c) in very low excitation nebulae the unobserved $\mathrm{Ar}^{+}$ions could dominate the argon abundance. The overall uncertainty related to the finally adopted argon abundances should be less than about $50 \%$, but it is likely to be more than $30 \%$.

Chlorine In only less than $25 \%$ of the sample could we determine the abundance of this element. The ionic lines generally detected in optical spectra are those of $\mathrm{Cl}^{+2}$, the predominate stage of ionization, at $\lambda 5517 \AA$ and $\lambda 5537 \AA$. They are often very weak. Due to the relatively large extinction in the direction of galactic center, the detections are biased to only those objects with high $\mathrm{Cl}$ abundances. Similar to $\mathrm{Ar}^{+3}$, the structure of $\mathrm{Cl}^{+2}$ is involving a $3 \mathrm{p}^{3}$-configuration. The ionic abundances could have thus influence from the existence of knots and concentrations in dense blobs. The expected errors in the total abunances are typically between $30 \%$ and $60 \%$.

There are ten nebulae in common with the sample studies by Aller \& Keyes (1987), and it is of interest to compare the results. While the spectral line intensities are in a fair agreement, the comparison of total abundances obtained in this work with those derived by Aller and Keyes shows that except for one case (PK $2-2.4)$ the overall agreement is, on average, better than $25 \%$. In the case of PK $2-2.4$ the discrepancies are about $40 \%$ to $50 \%$ and are likely due to the difference in the adopted density. Aller and Keyes adopted an electron density of $3000 \mathrm{~cm}^{-3}$, while in our case $11500 \mathrm{~cm}^{-3}$ is used. The radio continuum measurement by Gathier et al. (1983), however, suggests that the electron density should be much higher than $5000 \mathrm{~cm}^{-3}$, which supports our value, even if the nebulae is located at the distance of about $1 \mathrm{kpc}$ (the radial velocity of $+235 \mathrm{~km} \mathrm{~s}^{-1}$ strongly argues for the bulge membership). In Table 6 we list the bulge $\mathrm{PNe}$ studied by Aller \& Keyes (1987), which are not in common with those tabulated in Table 5 (see Sect. 2.3). The table is arranged in the same way as Table 5, except that the quality of the individual abundance determination is not presented. The overall quality of determination for these nebulae, as given in the original paper and scaled to the system used in this paper, is typically between $\mathrm{B}^{+}$and $B(0.20<\Delta \leq 0.60)$. We are now in a position to construct an evolutionary scenario to explain the abundances in planetary nebulae near the galactic centre. This is done in Paper II (Ratag et al. 1992).

Acknowledgements. One of us (MAR) acknowledges the financial support from the Overseas Fellowship Program (OFP) working under supervision of the Ministry of Research and Technology of Indonesia, and to the Indonesian National Institute of Aeronautics and Space (LAPAN).

\section{References}

Aaquist O.B., Kwok S., 1990, A\&AS 84, 229

Acker A.A., Chopinet M., Pottasch S.R., Stenholm B., 1987, A\&AS 71, 163

Acker A.A., Köppen J., Stenholm B., Jasniewicz G., 1989a, A\&AS 80, 201

Acker A.A., Stenholm B., Tylenda R., 1989b, A\&AS 77, 487 
Aitken D.K., Griffiths J., Jones B., 1976, MNRAS 176, 73P

Allen D.A., 1979, Observatory 99, 83

Aller L.H., 1956, "Gaseous Nebulae". Chapman and Hall, London

Aller L.H., 1984, "Physics of Thermal Gaseous Nebulae". Reidel, Dordrecht

Aller L.H., 1987, "Spectroscopy of Astrophysical Plasmas", Dalgarno A., Layzer D. (eds.). Cambridge Univ. Press, Cambridge, p. 89

Aller L.H., Czyzak S.J., 1983, ApJS 51, 211

Aller L.H., Keyes C.D., 1987, ApJS 65, 405

Balick B., 1975, ApJ 201, 705

Barker T., 1987a, ApJ 219, 914

Barker T., 1987b, ApJ 220, 193

Barker T., 1980, ApJ 240, 99

Bowen I.S., Wyse A.B., 1939, Lick Obs. Bull. 19, 1

Brocklehurst M., 1971, MNRAS 153, 471

Churchwell E., Smith L.F., Mathis J., Mezger P.G., Huchtmeier W., 1978, A\&A 70, 719

Churchwell E., Walmsley C.M., 1975, A\&A 38, 451

Clegg R.E.S., 1987, MNRAS 229, 31P

Clegg R.E.S., 1989, IAU Symp. 131, Torres-Peimbert S. (ed.). Kluwer, Dordrecht, p. 139

Clegg R.E.S., Middlemass D., 1987, MNRAS 228, 759

Diaz A.I., Tosi M., 1984, MNRAS 208, 365

Diaz A.I., Tosi M., 1986, A\&A 158, 60

Faúndez-Abans M., Maciel W.J., 1986, A\&A 158, 228

Feast M.W., 1968, MN 140, 345

Frogel J.A., Whitford A.E., 1987, ApJ 320, 199

Gathier R., Pottasch S.R., Goss W.M., van Gorkom J.H., 1983, A\&A 128,353

Gehren T., Nissen P.E., Kudritzki R.P., Butler K., 1985, "ESO Workshop on Production and Distribution of C, N, O Elements", Danziger I.J. et al. (eds.), p. 171

Güsten R., 1989, IAU Symp. 136, Morris M. (ed.). Kluwer, Dordrecht, p. 89

Harrington J.P., Seaton M.J., Adams S., Lutz J.H., 1982, MNRAS 199, 517

Husfeld D., Kudritzki R.P., Simon I.P., Clegg R.E.S., 1984, A\&A 134, 139

Janes K.A., 1979, ApJS 39, 135

Kaler J.B., 1986, ApJ 308, 322

Keyes C.D., Aller L.H., 1978, Ap\&SS 59, 91

Kinman T.D., Feast M.W., Lasker B.M., 1988, AJ 95, 804

Kurucz R.L., 1979, ApJS 40, 1

Leisy P., Dennefeld M., 1996, A\&AS 116, 95

Lester D.F., Bregman J.D., Witteborn F.C., Rank D.M., Dinerstein H.L., 1981, ApJ 248, 524

Luck R.E., 1982, ApJ 256, 177

Maciel W.J., Faúndez-Abans M., 1985, A\&A 149, 365

Matteucci F., Brocato E., 1990, ApJ 365, L539

Matteucci F., François P., 1989, MNRAS 239, 885

Mayor M., 1976, A\&A 48, 301

Mendoza C., 1983, IAU Symp. 103, Flower D. (ed.). Reidel, Dordrecht, p. 245

Milne D.K., Aller L.H., 1975, A\&A 38, 183

Milne D.K., Aller L.H., 1982, A\&AS 50, 209

Morris M., 1989, IAU Symp. 136, Morris M. (ed.). Kluwer Dordrecht, p. 171
Natta A., Pottasch S.R., Preite-Martinez A., 1980, A\&A 84, 284

Osterbrock D.E., 1984, "Astrophysics of Gaseous Nebulae". W.H. Freeman and Company, San Fransciso

Pagel B.E.J., 1979, "Stars and Star Systems", Westerlund B.E. (ed.). Reidel, Dordrecht p. 17

Peimbert M., Coster R., 1969, Bol. Obs. Tonantzintla y Tacubaya 5,3

Peimbert M., Torres-Peimbert S., 1977, MNRAS 179, 217

Peimbert M., Torres-Peimbert S., Rayo J.F., 1978, ApJ 220, 516

Peimbert M., Torres-Peimbert S., 1987, Rev. Mex. Astron. Astrofis. 14, 540

Perek L., Kohoutek L., 1967, "Catalogue of Galactic Planetary Nebulae", Czechoslovak Academy of Sciences, Prague

Pottasch S.R., 1984, Plan. Neb. Reidel, Dordrecht

Pottasch S.R., Preite-Martinez A., Olnon F.M., Mo Jing-Er., Kingma S., 1986, A\&A 161, 363

Pottasch S.R., Bignell C., Olling R., Zijlstra A.A., 1988, A\&A 205, 248

Price C.M., 1981, ApJ 247, 540

Ratag M.A., Pottasch S.R., 1990, A\&A 227, 207

Ratag M.A., Pottasch S.R., Zijlstra A.A., Menzies J., 1990, A\&A

Ratag M.A., Pottasch S.R., Dennefeld M., Menzies J.W., 1992, A\&A 255, 255 (Paper II)

Reid M.J., 1989, IAU Symp. 136, Morris M. (ed.). Kluwer, Dordrecht, p. 37

Rich R.M., 1986, Ph.D. Thesis, Caltech

Rich R.M., 1988, AJ 95, 828

Rocca-Volmerange B., Schaeffer R., 1990, A\&A 233, 427

Rubin R.H., 1986, ApJ 309, 334

Savage B.D., Mathis J.S., 1979, ARA\&A 17, 73

Schneider S.E., Terzian Y., Purgathofer A., Perinotto M., 1983, ApJS 52, 399

Seaton M.J., 1968, MNRAS 139, 129

Shaver P.A., McGee R.X., Newton L.M., Danks A.C., Pottasch S.R., 1983, MNRAS 204, 53

Shaw R.A., Kaler J.B., 1989, ApJS 69, 495

Shields G., Aller L.H., Keyes C.D., Czyzak S.J., 1981, ApJ 248, 569

Spitzer L. Jr., 1978, "Physical Processes in the Interstellar Medium". John Wiley and Sons, New York, p. 57-58

Terdrup D.M., 1988, AJ 96, 884

Torres-Peimbert S., Peimbert M., 1977, Rev. Mex. Astron. Astrof. 2, 181

Wannier P.G., 1989, IAU Symp. 136, Morris M. (ed.). Kluwer, Dordrecht, p. 107

Webster B.L., 1983 PASP 95, 610

Webster B.L., 1988, MNRAS 230, 377

Wyse A.B., 1942, ApJ 95, 356

Yorke H.W., 1988, "Radaiation in Moving Gaseous Media", $18^{\text {th }}$ Advances Course of the SAAS, Chmielewski Y. and Lanz T. (eds.)

Zeippen C.J., Butler K., Le Bourlot J., 1987, A\&A 188, 251

Zijlstra A.A., Pottasch S.R., Bignell C., 1989, A\&AS 79, 329

Zuckermann B., Aller L.H., 1986, ApJ 301, 772 